\title{
Group Generalized q-Rung Orthopair Fuzzy Soft Sets: New Aggregation Operators and Their Applications
}

\author{
Khizar Hayat $\left(\mathbb{D},{ }^{1}\right.$ Raja Aqib Shamim $\left(\mathbb{D},{ }^{1}\right.$ Hussain AlSalman $\left(\mathbb{D},{ }^{2}\right.$ Abdu Gumaei $(\mathbb{D}){ }^{3}$ \\ Xiao-Peng Yang $\mathbb{D}^{4},{ }^{4}$ and Muhammad Azeem Akbar $\mathbb{D D}^{5}$ \\ ${ }^{1}$ Department of Mathematics, University of Kotli, Kotli, Azad Jammu and Kashmir, Pakistan \\ ${ }^{2}$ Department of Computer Science, College of Computer and Information Sciences, King Saud University, \\ Riyadh 11543, Saudi Arabia \\ ${ }^{3}$ Computer Science Department, Faculty of Applied Sciences, Taiz University, Taiz 6803, Yemen \\ ${ }^{4}$ School of Mathematics and Statistics, Hanshan Normal University, Chaozhou 521041, China \\ ${ }^{5}$ Lappeenranta-Lahti University of Technology (LUT), Department of Software Engineering, Lappeenranta 53851, Finland
}

Correspondence should be addressed to Khizar Hayat; khizarhayat@uokajk.edu.pk and Abdu Gumaei; abdugumaei@gmail.com

Received 10 July 2021; Revised 21 November 2021; Accepted 25 November 2021; Published 31 December 2021

Academic Editor: Giuseppe D'Aniello

Copyright (C) 2021 Khizar Hayat et al. This is an open access article distributed under the Creative Commons Attribution License, which permits unrestricted use, distribution, and reproduction in any medium, provided the original work is properly cited.

In recent years, q-rung orthopair fuzzy sets have been appeared to deal with an increase in the value of $q>1$, which allows obtaining membership and nonmembership grades from a larger area. Practically, it covers those membership and nonmembership grades, which are not in the range of intuitionistic fuzzy sets. The hybrid form of q-rung orthopair fuzzy sets with soft sets have emerged as a useful framework in fuzzy mathematics and decision-makings. In this paper, we presented group generalized q-rung orthopair fuzzy soft sets (GGq-ROFSSs) by using the combination of q-rung orthopair fuzzy soft sets and q-rung orthopair fuzzy sets. We investigated some basic operations on GGq-ROFSSs. Notably, we initiated new averaging and geometric aggregation operators on GGq-ROFSSs and investigated their underlying properties. A multicriteria decision-making $(\mathrm{MCDM})$ framework is presented and validated through a numerical example. Finally, we showed the interconnection of our methodology with other existing methods.

\section{Introduction}

Zadeh originated the fuzzy set (FS) as an enlargement of the standard sets by the concept of inclusion of vague human judgements in computing situations [1]. The FS is indicated by the fuzzy information $\mu$, which gives values from the unit close interval $[0,1]$ for each prospector $x \in \mathscr{X}$. The idea of the FS plays an important role in the domain of soft computing, which manages vagueness, robustness, and partial truth. In some real-world difficulties where humanoid though attains reliable and unreliable information, the FS may not be sufficient to deal with underlying uncertainties.

In 1986, another shape of the FS called intuitionistic fuzzy sets (IFSs) was authorized by Atanassov, which provide a reliable grade $\mu(x)$ and unreliable grade $\nu(x)$ for all $x$ in the universe of discourse $X$. The IFSs are characterized by the sum $\mu(x)+\nu(x) \leq 1$ and the degree of indeterminacy $\pi(x)=1-\mu(x)-\nu(x)[2]$. Xu and Yager [3, 4] discussed the intuitionistic fuzzy value (IFV), which is an ordered pair of reliable and unreliable information for a component in the IFS on any $x$. Different rudiments of IFSs have been established such as aggregation operators [4], similarity and distance function $[5,6]$, and multicriteria decision-makings $(\mathrm{MCDM})$ [7]. The aggregation operators are imperious in the MCDM process, which attains a shape of the measurable information by the accumulation of big data [8-10].

The IFSs enhance FSs in a meaningful approach, which is more capable of overcoming uncertainties, sharpless boundaries caused by the hesitation, and lack of assurance in human cognition. Xu and Zhao [11] extended a meaningful and insightful view on the information synthesis for MCDM 
using IFSs. To deal with real-life cases of reliable and unreliable information, which do not satisfy inequality $\mu(x)+\nu(x) \leq 1$, Yager initiated Pythagorean fuzzy sets (PFSs) $[12,13]$ and q-rung orthopair fuzzy sets (q-ROFSs) [14], which are crucial generalizations of IFSs. The q-ROFSs possess overall anticipation of symmetry of reliable and unreliable information in a larger space [15], that is, $(\mu(x))^{q}+(\nu(x))^{q} \leq 1$. A q-ROFS appears as an IFS (PFS) when $q=1(q=2)$. The fundamental score function and operators for q-ROFSs were investigated by Liu and Wang [16]. Several basic properties of PFSs and q-ROFSs can be seen in the literature [17-21]. Notably, researchers around the planet check out hybrid MCDM methods of PFSs and q-ROFSs using TODIM [22], TOPSIS [23, 24], MULTIMOORA [25], MABAC method [26], aggregation operators $[17,27-33]$, entropy measures [34], and distance measures [35].

A general parametrization model called soft set theory initiated by Molodtsov [36] has a great tendency to cop uncertainties. The soft set is free of inadequacy as it is a classical tool for coping parameters. It is further connected with usual mathematical operations on sets by Maji et al. [37] and Ali et al. [38]. A combination of soft sets and FSs known as fuzzy soft sets was introduced by Maji et al. [39], and it has been applied in various fields [40-46]. An extended form of fuzzy soft sets, known as intuitionistic fuzzy soft sets (IFSSs), was initiated by Maji et al. [47]. Recently, q-rung orthopair fuzzy soft sets (q-ROFSSs) have been introduced by Hamid et al. [48]. The model of q-ROFSS is a valuable tool to deal with vagueness by means of the label of parameters along with reliable and unreliable grades in the larger space [49]. Hussain et al. [50] presented MCDM techniques using averaging operators on q-ROFSSs. The generalized IFSSs (GIFSSs) were investigated by Agarwal et al. [51], and it possesses an important opinion with the model IFSS. A different scenario that overcomes the adequacies [52] of the original concept of GIFSSs was given by Feng et al. [53]. Both the ideas of GIFSSs were extended by several researchers Garg and Arora [54], Hayat et al. [55, 56], and Khan et al. [57, 58]. GGIFSSs produce a deep and meaningful insight in the MCDM problem by merging aggregation operators [56]. Another aspect of GGIFSS-based operators has been investigated by Hayat et al. [59], which handle information in a collected form. On the prospect of group-based GIFSSs (GGIFSSs) [56, 59], it is required to develop underlying operators, which can handle MCDM problems in different scenarios of combinations of information. More importantly, the extended space of q-ROFSs is the general form to deal with any implicit information.

On this prospect, there is a huge capacity to exercise another view of GGIFSS aggregation operators because the q-ROFSs relays the ambiguous information in higher productive ways than the GGIFSSs. Another important and fundamental point is to develop a different study to GGIFSSs that aggregate information concerning attributes until final ranking appears. Thus, we developed the group-based generalized q-ROFSSs (GGq-ROFSSs) and new aggregation operators through entire components in GGq-ROFSSs. By motivations of the above discussion, the purpose and aim of this article are given as follows:

(1) To initiate a different form of aggregation operators for GGq-ROFSSs that do not abandon the importance of attributes initially and do not quickly fascinate alternatives

(2) To develop an internal mechanism that gently addresses the importance of parameters in aggregation operators for GGq-ROFSSs

(3) To address the higher range of reliable and unreliable information in GGq-ROFSSs for possible values of $q$

(4) To develop the MCDM method for GGq-ROFSSs environment

In Section 2, we recall basic ideas of IFSs, PFSs, q-ROFSs, soft sets, and q-ROFSSs. In Sections 3 and 4, we discuss the notions of GGq-ROFSSs and their operations. In Section 5, we define new aggregations operators on GGq-ROFSSs. In Section 6, we give a new method of MCDM and a numerical example of real-life applications. Section 7 gives comparisons with other existing methods, and the last section concludes the paper.

\section{Preliminaries}

In this section, we will recall the concepts of IFSs, PFSs, q-ROFSs, soft sets, and q-ROFSSs. Throughout this section, $\mathscr{X}$ will represent the collection of alternatives.

2.1. Intuitionistic Fuzzy Sets and Pythagorean Fuzzy Sets. A FS is a mapping $\mu: \mathscr{X} \longrightarrow[0,1]$, where $\mu$ is membership grade for an element $g \in \mathscr{X}$ [1]. In several real-life situations, reliable and unreliable information rectifies the proper signification of uncertainties. FSs were not sufficient in such situations; therefore, the concept of IFS was introduced.

Definition 1 (see [2]). An IFS $\mathscr{F}$ is expressed as follows:

$$
\mathscr{F}=\{(g, \mu(g), \nu(g)) \mid g \in \mathscr{X}\} .
$$

With the functions $\mu: \mathscr{X} \longrightarrow[0,1], \nu: \mathscr{X} \longrightarrow[0,1]$ called reliable and unreliable grades of an element $g$ of $\mathscr{X}$ under the condition that the following inequality holds:

$$
0 \leq \mu(g)+\nu(g) \leq 1 .
$$

For an element $g \in \mathscr{X},\langle\mu, \nu\rangle$ is called IF value (IFV) in $\mathscr{F}$. In an IFS, the hesitancy of an IFV $\langle\mu, \nu\rangle$ to $\mathscr{F}$ is given by

$$
\pi_{\mathscr{F}}=1-\mu(g)-\nu(g) .
$$

The hesitancy of IFV is also called indeterminacy of the $g \in \mathscr{X}$ in $\mathscr{F}$.

Definition 2 (see $[12,13])$. A PFS $\mathscr{F}$ is expressed as follows:

$$
\mathscr{P}=\{(g, \mu(g), \nu(g)) \mid g \in \mathscr{X}\} .
$$


With the functions $\mu: X \longrightarrow[0,1], v: \mathscr{X} \longrightarrow[0,1]$ called reliable and unreliable grades of an element $g$ of $\mathscr{X}$ under the condition that the following inequality holds:

$$
0 \leq(\mu(g))^{2}+(\nu(g))^{2} \leq 1 .
$$

For an element $g \in \mathscr{X},\langle\mu, \nu\rangle$ is called PF value (PFV) in $\mathscr{P}$. In an PFS, the hesitancy (or indeterminacy) of an PFV $\langle\mu, \nu\rangle$ to $\mathscr{P}$ is given by

$$
\pi_{\mathscr{P}}=\sqrt{1-(\mu(g))^{2}-(\nu(g))^{2}} .
$$

2.2. q-Rung Orthopair Fuzzy Sets. In 2016, Yager extended the range of double-graded fuzzy models in higher space. It is defined as

Definition 3 (see [14]). A q-RFS $\mathscr{R}$ is defined as

$$
\mathscr{R}=\{(g, \mu(g), \nu(g)) \mid g \in \mathscr{X}\} .
$$

With the functions $\mu: X \longrightarrow[0,1], v: \mathscr{X} \longrightarrow[0,1]$ called reliable and unreliable grades of an element $g$ of $\mathscr{X}$ under the condition that the following inequality holds:

$$
0 \leq(\mu(g))^{q}+(\nu(g))^{q} \leq 1 .
$$

Particularly, the hesitancy degree for q-ROFS is given as

$$
\pi_{\mathscr{R}}=\sqrt[q]{1-(\mu(g))^{2}-(\nu(g))^{2}} .
$$

The pair $\langle\mu, \nu\rangle$ is called q-rung orthopair fuzzy value (qROFV) for an object $g \in \mathscr{X}$. Let $\mathscr{R}_{1}=\{(g, \mu(g)$, $v(g)) \mid g \in \mathscr{X}\}$ and $\mathscr{R}_{2}=\{(g, s(g), t(g)) \mid g \in \mathscr{X}\}$ be two q-ROFSs; then,

$$
\begin{aligned}
& \mathscr{R}_{1} \cup \mathscr{R}_{2}=\{(g, \max \{\mu(g), s(g)\}, \min \{\nu(g), t(g)\}) \mid g \in \mathscr{X}\}, \\
& \mathscr{R}_{1} \cap \mathscr{R}_{2}=\{(g, \min \{\mu(g), s(g)\}, \max \{\nu(g), t(g)\}) \mid g \in \mathscr{X}\}, \\
& \mathscr{R}_{1} \subseteq \mathscr{R}_{2} \Leftrightarrow\{\mu(g) \leq s(g), \nu(g) \leq t(g) \forall g \in \mathscr{X}\}, \\
&\left(\mathscr{R}_{1}\right)^{c}=\{(g, \nu(g), \mu(g)) \mid g \in \mathscr{X}\} .
\end{aligned}
$$

The study on q-ROFS is extended by Liu and Wang [16] in the following crucial notions:

Definition 4 (see [16]). Let $b=\langle\mu, \nu\rangle$ be q-ROFN; then, the score function is defined as follows:

$$
S_{\text {liu }}(b)=\mu^{q}-\nu^{q} .
$$

This notion is effective when we have to transfer a q-ROFN to a real value in interval $[-1,1]$ and therefore we can compare two or more q-ROFNs on their score functions. Consider a special case when $b_{1}=\left\langle\mu_{1}, v_{1}\right\rangle, b_{2}=\left\langle\mu_{2}, v_{2}\right\rangle$ with the condition $\mu_{i}=v_{i} \forall i=1,2$. Then, $s\left(b_{1}\right)=0=s\left(b_{2}\right)$; thus, $S_{\text {liu }}$ has an inadequacy for such a case. Therefore, in this study, when $S_{\text {liu }}$ fails, we will use the following.

Definition 5 (see [32]). Let $b=\langle\mu, \nu\rangle$ be q-ROFN; then,

$$
S_{p x}(b)=\left(\frac{e^{\mu^{q}-v^{q}}}{e^{\mu^{q}-v^{q}}+1}-\frac{1}{2}\right) \pi^{q}+\mu^{q}-v^{q} .
$$

Definition 6 (see [16]). Let us take the collection of q-ROFNs $b_{k^{\prime}}=\left(\mu_{k^{\prime}}, v_{k^{\prime}}\right), \quad k^{\prime}=1,2,3, \ldots, n$ and weight vector $\left[\lambda_{1}, \lambda_{2}, \ldots, \lambda_{n}\right]$; then, the q-rung orthopair fuzzy weighted averaging operator (q-ROFWA) is indicated as

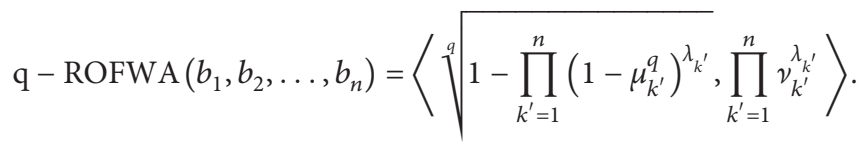

Definition 7 (see [16]). Let us take the collection of q-ROFNs $b_{k^{\prime}}=\left(\mu_{k^{\prime}}, v_{k^{\prime}}\right), k^{\prime}=1,2,3, \ldots, n$ and weight vector $\left[\lambda_{1}, \lambda_{2}\right.$, $\left.\ldots, \lambda_{n}\right]$; then, the q-rung orthopair fuzzy weighted geometric operator (q-ROFWG) is indicated as

$$
\mathrm{q}-\operatorname{ROFWG}\left(b_{1}, b_{2}, \ldots, b_{n}\right)=\left\langle\prod_{k^{\prime}=1}^{n} \mu_{k^{\prime}}^{\lambda_{k^{\prime}}}, \sqrt[q]{1-\prod_{k^{\prime}=1}^{n}\left(1-v_{k^{\prime}}^{q}\right)^{\lambda_{k^{\prime}}}}\right\rangle .
$$

The q-ROFWA and q-ROFWG are effective to aggregate data involving large number of $\mathrm{q}$-ROFNs to a single q-ROFN.

2.3.q-Rung Orthopair Fuzzy Soft Sets. Jointly the approach of soft sets [36] with q-ROFSs is known as the q-rung orthopair fuzzy soft set, which is handy in MCDM problems. The notion of soft set is described as follows.

Definition 8 (see [36]). Let $\mathscr{X}$ be a fixed set and $P(\mathscr{X})$ be the set of all subsets of $\mathscr{X}$. Consider the set of parameters $E$ and $\mathscr{A}$ be the subset of $E$. Let us define a function $\delta$ as

$$
\delta: \mathscr{A} \longrightarrow P(\mathscr{X}) \text {. }
$$

Then, pair $(\mathcal{S}, \mathscr{A})=\{(g, \mathcal{S}(g)) \mid g \in \mathscr{A}, \mathcal{S}(g) \in P(\mathscr{X})\}$ is called the soft set.

Definition 9 (see [49]). Let $(\mathscr{X}, E)$ be a soft universe and $\mathscr{A} \subseteq E$. Define a mapping $Q: \mathscr{A} \longrightarrow \mathrm{q}-\operatorname{ROFS}(\mathscr{X})$; then, pair $(\mathscr{Q}, \mathscr{A})$ is called the q-rung orthopair fuzzy soft set (qROFSS) over $\mathscr{X}$, where $\mathrm{q}-\mathrm{ROFS}(\mathscr{X})$ denotes the collection of all q-ROFSs over $\mathscr{X}$. The q-ROFSS $(\mathscr{Q}, \mathscr{A})$ can be described as

$$
\begin{aligned}
(\mathscr{Q}, \mathscr{A}) & =\{(e,\{\rho,\langle\mu(\rho), \nu(\rho)\rangle\}) \mid e \in \mathscr{A}, \rho \in \mathscr{X}\}, \\
\operatorname{or}(\mathscr{Q}, \mathscr{A}) & =\left\{\left(e,\left\{\frac{\rho}{\langle\mu(\rho), \nu(\rho)\rangle}\right\}\right) \mid e \in \mathscr{A}, \rho \in \mathscr{X}\right\},
\end{aligned}
$$

where $\mu(\rho): X \longrightarrow[0,1]$ and $\nu(\rho): \mathscr{X} \longrightarrow[0,1]$ are reliable and nonreliable grades of $\rho$ fulfilling inequality $0 \leq \mu^{q}(\rho)+\nu^{q}(\rho) \leq 1$. Take $\mu_{i j}=\mu_{e_{j}}\left(\rho_{i}\right), v_{i j}=\nu_{e_{j}}\left(\rho_{i}\right)$, where $i=1,2, \ldots, m, j=1,2, \ldots, n$. Then, the tabular form of $\mathrm{q}-\operatorname{ROFSS}(\mathscr{Q}, \mathscr{A})$ is given in Table 1 . 
TABle 1: q-ROFSS $(\mathscr{Q}, \mathscr{A})$.

\begin{tabular}{ccccc}
\hline $\mathscr{X} \mid \mathscr{A}$ & $e_{1}$ & $e_{2}$ & $\cdots$ & $e_{n}$ \\
\hline$\rho_{1}$ & $\left\langle\mu_{11}, v_{11}\right\rangle$ & $\left\langle\mu_{12}, v_{12}\right\rangle$ & $\cdots$ & $\left\langle\mu_{1 n}, v_{1 n}\right\rangle$ \\
$\rho_{2}$ & $\left\langle\mu_{21}, v_{21}\right\rangle$ & $\left\langle\mu_{22}, v_{22}\right\rangle$ & $\ldots$ & $\left\langle\mu_{2 n}, v_{2 n}\right\rangle$ \\
$\vdots$ & $\vdots$ & $\vdots$ & $\ddots$ & $\vdots$ \\
$\rho_{m}$ & $\left\langle\mu_{m 1}, v_{m 1}\right\rangle$ & $\left\langle\mu_{m 2}, v_{m 2}\right\rangle$ & $\cdots$ & $\left\langle\mu_{m n}, v_{m n}\right\rangle$ \\
\hline
\end{tabular}

For simplicity, we will represent $(\mathscr{Q}, \mathscr{A})$ by $\mathscr{Q}_{\mathscr{A}}$.

Definition 10 (see [49]). Consider two q-ROFSSs $\mathbb{Q}_{\mathscr{A}_{1}}$ and $\mathcal{Q}_{\mathscr{A}_{2}^{\prime}}$ on $\mathscr{X}$. We say $\mathcal{Q}_{\mathscr{A}_{1}} \subseteq \widehat{Q}_{\mathscr{A}_{2}^{\prime}}^{\prime}$ if and only if

(i) $\mathscr{A}_{1} \subseteq \mathscr{A}_{2}$

(ii) $Q(e)$ is q-ROF subset of $Q^{\prime}(e)$ for all $e \in \mathscr{A}_{1} \subseteq \mathscr{A}_{2}$

Definition 11 (see [49]). Consider two q-ROFSSs $\mathscr{Q}_{\mathscr{A}_{1}^{\prime}}^{\prime}$ and

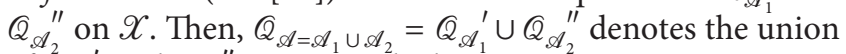
of $\mathscr{Q}_{\mathscr{A}_{1}^{\prime}}^{\prime}$ and $\mathbb{Q}_{\mathscr{A}_{2}}^{\prime \prime}$ on $\mathscr{X}$, such that

$$
Q(e)= \begin{cases}Q^{\prime}(e), & \text { if } e \in \mathscr{A}_{1}-\mathscr{A}_{2}, \\ \mathcal{Q}^{\prime \prime}(e), & \text { if } e \in \mathscr{A}_{2}-\mathscr{A}_{1}, \\ Q^{\prime}(e) \cup \mathcal{Q}^{\prime \prime}(e), & \text { if } e \in \mathscr{A}_{1} \cap \mathscr{A}_{2},\end{cases}
$$

where $\mathbb{Q}^{\prime}(e) \cup Q^{\prime \prime}(e)$ is the union of q-ROFSs $Q^{\prime}(e), Q^{\prime \prime}(e)$.

Definition 12 (see [49]). Consider two q-ROFSSs $\mathcal{Q}_{\mathscr{A}_{1}^{\prime}}$ and

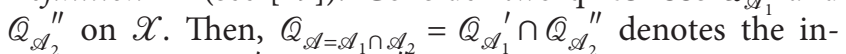
tersection of $\mathscr{Q}_{\mathscr{A}_{1}}^{\prime}$ and $\mathscr{Q}_{\mathscr{A}_{2}}^{\prime \prime 2}$ on $\mathscr{X}$, such that $Q(e)=Q^{\prime}$ $(e) \cap Q^{\prime \prime}(e)$, where $e \in \mathscr{A}_{1} \cap \mathscr{A}_{2} \neq \varnothing$, where $Q^{\prime}(e) \cap \mathbb{Q}^{\prime \prime}(e)$ is the intersection of q-ROFSs $Q^{\prime}(e), Q^{\prime \prime}(e)$.

\section{Group Generalized q-Rung Orthopair Fuzzy Soft Sets}

In this section, we will define generalized q-Rung orthopair fuzzy soft sets (Gq-ROFSSs) and group-based generalized q-Rung orthopair fuzzy soft sets (GGq-ROFSSs). First GqROFSS is described as follows.

Definition 13. Consider a soft universe $(\mathscr{X}, E)$ and $\mathscr{A}$ contained in $E$. A triple $(\mathscr{Q}, \mathscr{A}, \alpha)$ is called Gq-ROFSS over $\mathscr{X}$ if $(\mathscr{Q}, \mathscr{A})$ is a q-ROFSS over $\mathscr{X}$ and $\alpha$ is a q-ROFS over $\mathscr{A}$.

In Gq-ROFSS, only one extra opinion $\alpha$ appears, but in many real-life satisfactions, more than one crucial additional opinions are needed. Thus, we define a greater prospect of Gq-ROFSS as GGq-ROFSS as follows.

Definition 14. Consider a soft universe $(\mathscr{X}, E)$ and $\mathscr{A}$ contained in $E$. A triple $(\mathscr{Q}, \mathscr{A}, \eta)$ is called GGq-ROFSS over $\mathscr{X}$ if $(\mathscr{Q}, \mathscr{A})$ is a q-ROFSS over $\mathscr{X}$ and $\eta=\left\{\alpha_{1}, \alpha_{2}, \ldots, \alpha_{p}\right\}$, where $\alpha_{1}, \alpha_{2}, \ldots, \alpha_{p}$ are the parametrized q-ROFSs (PqROFSs) over $\mathscr{A}$. In other words, $\eta$ is the group Pq-ROFSs considered by " $p$ " number of senior experts/moderators.

Remark 1. If $p=1$ in GGq-ROFSSs, then it is Gq-ROFSS. Therefore, Gq-ROFSS is a special case of GGq-ROFSS, and thus, generally, we will focus on GGq-ROFSSs.
A broaden tabular form of GGq-ROFSS (in Definition 14) is given in Table 2.

In Table 2, the light gray part represents q-ROFSS and the brown part represents the group of q-ROFSs in GGqROFSS.

\section{Operations on Group-Based Generalized q-Rung Orthopair Fuzzy Soft Sets}

In this section, we will define subset, union, intersection, and complement of GGq-ROFSSs.

Definition 15. Consider a soft universe $(\mathscr{X}, E)$ and $\mathscr{A}, \mathscr{B}$ contained in $E$. Let two GGq-ROFSSs $\left(Q_{1}, \mathscr{A}_{1}, \eta_{1}\right)$ and $\left(Q_{2}\right.$, $\left.\mathscr{A}_{2}, \eta_{2}\right)$ on $\mathscr{X}$, where $\mathscr{A} \subseteq \mathscr{B}, \eta_{1}=\left\{\alpha_{1}, \alpha_{2}, \ldots, \alpha_{p}\right\}, \eta_{2}=\left\{\beta_{1}\right.$, $\left.\beta_{2}, \ldots, \beta_{p}\right\}$, respectively, are group Pq-ROFSs related to " $p$ " number of senior experts/moderators. The $\eta_{1}$ is group q-ROF subset of $\eta_{1}$ if and only if $s_{\alpha_{1}}\left(e_{k}\right) \leq s_{\beta_{1}}\left(e_{k}\right), s_{\alpha_{2}}\left(e_{k}\right)$ $\leq s_{\beta_{2}}\left(e_{k}\right), \ldots, s_{\alpha_{p}}\left(e_{k}\right) \leq s_{\beta_{p}}\left(e_{k}\right)$, and $t_{\alpha_{1}}\left(e_{k}\right) \leq t_{\beta_{1}}\left(e_{k}\right), t_{\alpha_{2}}$ $\left(e_{k}\right) \leq t_{\beta_{2}}\left(e_{k}\right), \ldots, t_{\alpha_{p}}\left(e_{k}\right) \leq t_{\beta_{p}}\left(e_{k}\right)$ and for each $e_{k} \in \mathscr{A}$. It is denoted as $\eta_{1} \Subset \eta_{2}$.

In the prospect of Definition 15, we introduce GGqROFS subsets.

Definition 16. Let two GGq-ROFSS $\left(\mathscr{Q}_{1}, \mathscr{A}_{1}, \eta_{1}\right)$ and $\left(\mathscr{Q}_{2}, \mathscr{A}_{2}, \eta_{2}\right)$ on $\mathscr{X}$, where $\mathscr{A}, \mathscr{B} \subset E$. Then, $\left(\mathscr{Q}_{1}, \mathscr{A}_{1}, \eta_{1}\right)$ is a GGq-ROFS subset of $\left(\mathscr{Q}_{2}, \mathscr{A}_{2}, \eta_{2}\right)$ if

(i) $\left(Q_{1}, \mathscr{A}_{1}\right) \subseteq\left(\mathscr{Q}_{2}, \mathscr{A}_{2}\right)$

(ii) $\eta_{1} \Subset \eta_{2}$

Definition 17. Let two GGq-ROFSSs $\left(Q_{1}, \mathscr{A}_{1}, \eta_{1}\right)$ and $\left(\mathscr{Q}_{2}, \mathscr{A}_{2}, \eta_{2}\right)$ on $\mathscr{X}$, where $\mathscr{A}, \mathscr{B} \subset E$ such that $\mathscr{A}_{1} \cup \mathscr{A}_{2}=\mathscr{C}$ and $\eta_{1}=\left\{\alpha_{1}, \alpha_{2}, \ldots, \alpha_{p}\right\}, \eta_{2}=\left\{\beta_{1}, \beta_{2}, \ldots, \beta_{p}\right\}$. The extended union of $\left(Q_{1}, \mathscr{A}_{1}, \eta_{1}\right)$ and $\left(\mathbb{Q}_{2}, \mathscr{A}_{2}, \eta_{2}\right)$ is investigated in the form of GGq-ROFSS. which is given as follows:

$$
(\mathbb{Q}, \mathscr{C}, \eta)=\left(\mathscr{Q}_{1}, \mathscr{A}_{1}, \eta_{1}\right) \cup_{e}\left(\mathscr{Q}_{2}, \mathscr{A}_{2}, \eta_{2}\right),
$$

such that

(i) $(\mathbb{Q}, \mathscr{C})=\left(\mathscr{Q}_{1}, \mathscr{A}_{1}\right) \cup_{e}\left(\mathscr{Q}_{2}, \mathscr{A}_{2}\right)$. (ii) For each $e \in \mathscr{C},\left\langle\mu_{\gamma_{i^{\prime}}}, v_{\gamma_{i^{\prime}}}\right\rangle i^{\prime}=1,2, \ldots, p$ is defined

$$
\begin{aligned}
& \mu_{\gamma_{i^{\prime}}}(e)= \begin{cases}\mu_{\alpha_{i^{\prime}}}(e), & \text { if } e \in \mathscr{A}_{1}-\mathscr{A}_{2} ; \\
\mu_{\beta_{i^{\prime}}}(e), & \text { if } e \in \mathscr{A}_{2}-\mathscr{A}_{1} ; \\
\max \left\{\mu_{\alpha_{i^{\prime}}}(e), \mu_{\beta_{i^{\prime}}}(e)\right\}, & \text { if } e \in \mathscr{A}_{1} \cap \mathscr{A}_{2},\end{cases} \\
& v_{\gamma_{i^{\prime}}}(e)= \begin{cases}v_{\alpha_{i^{\prime}}}(e), & \text { if } e \in \mathscr{A}_{1}-\mathscr{A}_{2} ; \\
v_{\beta_{i^{\prime}}}(e), & \text { if } e \in \mathscr{A}_{2}-\mathscr{A}_{1} ; \\
\min \left\{v_{\alpha_{i^{\prime}}}(e), v_{\beta_{i^{\prime}}}(e)\right\}, & \text { if } e \in \mathscr{A}_{1} \cap \mathscr{A}_{2},\end{cases}
\end{aligned}
$$

where $\eta=\left\{\gamma_{1}, \gamma_{2}, \ldots, \gamma_{p}\right\}$. 
Table 2: GGq-ROFSS $(\mathscr{Q}, \mathscr{A}, \eta)$.

\begin{tabular}{lcccc}
\hline$\chi \mid \mathscr{A}$ & $e_{1}$ & $e_{2}$ & $\ldots$ & $e_{n}$ \\
\hline$\rho_{1}$ & $\left\langle\mu_{11}, v_{11}\right\rangle$ & $\left\langle\mu_{12}, v_{12}\right\rangle$ & $\ldots$ & $\left\langle\mu_{1 n}, v_{1 n}\right\rangle$ \\
$\rho_{2}$ & $\left\langle\mu_{21}, v_{21}\right\rangle$ & $\left\langle\mu_{22}, v_{22}\right\rangle$ & $\ldots$ & $\left\langle\mu_{2 n}, v_{2 n}\right\rangle$ \\
$\vdots$ & $\vdots$ & $\vdots$ & $\ddots$ & $\vdots$ \\
$\rho_{\mathrm{m}}$ & $\left\langle\mu_{m 1}, v_{m 1}\right\rangle$ & $\left\langle\mu_{m 2}, v_{m 2}\right\rangle$ & $\ldots$ & $\left\langle\mu_{m n}, v_{m n}\right\rangle$ \\
\hline$\alpha_{1}$ & $\left\langle s_{11}, t_{11}\right\rangle$ & $\left\langle s_{12}, t_{12}\right\rangle$ & $\ldots$ & $\left\langle s_{1 n}, t_{1 n}\right\rangle$ \\
$\alpha_{2}$ & $\left\langle s_{21}, t_{21}\right\rangle$ & $\left\langle s_{22}, t_{22}\right\rangle$ & $\ldots$ & $\left\langle s_{2 n}, t_{2 n}\right\rangle$ \\
$\vdots$ & $\vdots$ & $\vdots$ & $\ddots$ & $\vdots$ \\
$\alpha_{\mathrm{p}}$ & $\left\langle s_{p 1}, t_{p 1}\right\rangle$ & $\left\langle s_{p 2}, t_{p 2}\right\rangle$ & $\ldots$ & $\left\langle s_{p n}, t_{p n}\right\rangle$ \\
\hline
\end{tabular}

Definition 18. Let two GGq-ROFSSs be $\left(\mathscr{Q}_{1}, \mathscr{A}_{1}, \eta_{1}\right)$ and $\left(\mathscr{Q}_{2}, \mathscr{A}_{2}, \eta_{2}\right)$ on $\mathscr{X}$, where $\mathscr{A}, \mathscr{B} \subset E$ such that $\mathscr{A}_{1} \cap \mathscr{A}_{2}=\mathscr{C}$ and $\eta_{1}=\left\{\alpha_{1}, \alpha_{2}, \ldots, \alpha_{p}\right\}, \eta_{2}=\left\{\beta_{1}, \beta_{2}, \ldots, \beta_{p}\right\}$. The restricted intersection of $\left(\mathscr{Q}_{1}, \mathscr{A}_{1}, \eta_{1}\right)$ and $\left(\mathscr{Q}_{2}, \mathscr{A}_{2}, \eta_{2}\right)$ is investigated in the form of GGq-ROFSS, which is given as

$$
(\mathbb{Q}, \mathscr{C}, \eta)=\left(\mathscr{Q}_{1}, \mathscr{A}_{1}, \eta_{1}\right) \cap_{r}\left(\mathscr{Q}_{2}, \mathscr{A}_{2}, \eta_{2}\right) \text {, }
$$

such that

(i) $(\mathbb{Q}, \mathscr{C})=\left(\mathscr{Q}_{1}, \mathscr{A}_{1}\right) \cap_{r}\left(\mathscr{Q}_{2}, \mathscr{A}_{2}\right)$

(ii) For each $e \in \mathscr{C},\left\langle\mu_{\gamma_{i^{\prime}}}, v_{\gamma_{i^{\prime}}}\right\rangle i^{\prime}=1,2, \ldots, p$ is defined as $\mu_{\gamma_{i^{\prime}}}(e)=\min \left\{\mu_{\alpha_{i^{\prime}}}(e), \mu_{\beta_{i^{\prime}}}(e)\right\}$ and $\nu_{\gamma_{i^{\prime}}}(e)=\max$ $\left\{v_{\alpha_{i^{\prime}}}(e), v_{\beta_{i^{\prime}}}(e)\right\}$ for all $e \in \mathscr{A}_{1} \cap \mathscr{A}_{2}$, where $\eta=\left\{\gamma_{1}\right.$, $\left.\gamma_{2}, \ldots, \gamma_{p}\right\}$

Now, we will take an example of GGq-ROFSS to clarify the above concepts.

Example 1. Let $\mathscr{X}=\left\{\rho_{1}, \rho_{2}, \rho_{3}, \rho_{4}, \rho_{5}\right\}$ be the universal set consisting of five different kinds of face masks available in market, and the set of attributes $E$ is given as $E=\left\{e_{1}, e_{2}\right.$, $\left.e_{3}, e_{4}, e_{5}\right\}$, where each $e_{i}$, respectively, stands for affordable price, good fabrication, effective comfortable design, capable of stopping viruses and bacteria, and comfortable breathing while wearing the mask. Let the two buyers $\mathscr{T}_{1}$ and $\mathscr{T}_{2}$, respectively, have the following preferences while buying the face mask:

$$
\begin{aligned}
& \mathscr{A}=\left\{e_{1}, e_{4}\right\}, \\
& \mathscr{B}=\left\{e_{2}, e_{4}, e_{5}\right\} \subset E .
\end{aligned}
$$

The GGq-ROFSSs $(\mathscr{Q}, \mathscr{A}, \eta)$ and $\left(\mathscr{Q} \prime, \mathscr{B}, \eta^{\prime}\right)$ for buyers $\mathscr{T}_{1}$ and $\mathscr{T}_{2}$ are interpreted in Tables 3 and 4 , where two senior persons $h_{1}$ and $h_{2}$ provide their opinions on q-ROFSSs (given in the light gray parts of Tables 3 and 4). The extra inputs as the group of q-ROFSs of $h_{1}$ and $h_{2}$ are interpreted in the brown parts of both the tables. The union and intersection of $(\mathscr{Q}, \mathscr{A}, \eta)$ and $\left(\mathscr{Q}^{\prime}, \mathscr{B}, \eta^{\prime}\right)$ are computed in Tables 5 and 6 respectively.
Definition 19. Consider a soft universe $(\mathscr{X}, E)$ and $\mathscr{A}$ contained in $E$. Let GGq-ROFSS be $(\mathscr{Q}, \mathscr{A}, \eta)$ on $\mathscr{X}$ where $\eta=\left\{\alpha_{1}, \alpha_{2}, \ldots, \alpha_{p}\right\}$. The complement of $(\mathscr{Q}, \mathscr{A}, \eta)$ is denoted by $\left(\mathscr{Q}^{c}, \mathscr{A}, \eta^{c}\right)$, where $\left(\mathscr{Q}^{c}, \mathscr{A}\right)$ is the complement of q-ROFSS $(\mathscr{Q}, \mathscr{A})$ and $\eta^{c}=\left\{\alpha_{1}^{c}, \alpha_{2}^{c}, \ldots, \alpha_{p}^{c}\right\}$.

Definition 20. Let GGq-ROFSS be $(\mathscr{Q}, \mathscr{A}, \eta)$ given in Table 2.

(1) If $>\mu_{j k}, v_{j k}<=>1,0<$ and $>s_{i^{\prime} k}, t_{i^{\prime} k}<=>1,0<$ for all $j=1,2, \ldots, m, k=1,2, \ldots, n$ and $i^{\prime}=1,2, \ldots, p$, then $(\mathscr{Q}, \mathscr{A}, \eta)$ is called whole GGq-ROFSS. It is denoted by $\mathscr{W} \mathscr{A}$.

(2) If $>\mu_{j k}, v_{j k}<=>0,1<$ and $>s_{i^{\prime} k}, t_{i^{\prime} k}<=>0,1<$ for all $j=1,2, \ldots, m, k=1,2, \ldots, n$ and $i^{\prime}=1,2, \ldots, p$, then $(\mathbb{Q}, \mathscr{A}, \eta)$ is called null GGq-ROFSS. It is denoted by $\mathcal{N}_{\mathscr{A}}$.

Proposition 1. Let $Q_{\mathscr{A}}^{\eta}=(\mathscr{Q}, \mathscr{A}, \eta)$ be a $G G q$-ROFSS over $\mathscr{X}$. Then,

(1) $Q_{\mathscr{A}}^{\eta} \cup \mathbb{Q}_{\mathscr{A}}^{\eta}=Q_{\mathscr{A}}^{\eta}, Q_{\mathscr{A}}^{\eta} \cap Q_{\mathscr{A}}^{\eta}=Q_{\mathscr{A}}^{\eta}$

(2) $Q_{\mathscr{A}}^{\eta} \cup \mathscr{W}_{\mathscr{A}}=\mathscr{W}_{\mathscr{A}}, \mathbb{Q}_{\mathscr{A}}^{\eta} \cap \mathscr{W}_{\mathscr{A}}=\mathbb{Q}_{\mathscr{A}}^{\eta}$

(3) $Q_{\mathscr{A}}^{\eta} \cap \mathscr{N}_{\mathscr{A}}=\mathcal{N}_{\mathscr{A}}, Q_{\mathscr{A}}^{\eta} \cup \mathcal{N}_{\mathscr{A}}=Q_{\mathscr{A}}^{\eta}$

\section{Aggregation Operators on GGq-ROFSS}

To attain substantial effect, there is an immense need to define better aggregation operators that accurately deal with all components of GGq-ROFSSs.

\subsection{GWq-ROFW Operators}

Definition 21. Consider Definition 14, where GGq-ROFSS $(\mathbb{Q}, \mathscr{A}, \eta)$ is given in Table 2 , where the light gray part represents q-ROFSS and the brown part describes the group of q-ROFSs of " $p$ " number of senior moderators. Let $\left\langle\mu_{0 k}^{j}, v_{0 k}^{j}\right\rangle=\left\langle\mu_{j k}, v_{j k}\right\rangle, j=1,2, \ldots, n, k=1,2, \ldots, m$, that is to say $\left\langle\mu_{0 k}^{j}, v_{0 k}^{j}\right\rangle$ are q-ROFVs in the light gray part of Table 2. Moreover, $\left\langle\mu_{i^{\prime} k^{\prime}}^{j}, v_{i^{\prime} k}^{j}\right\rangle=\left\langle s_{i^{\prime} k}, t_{i^{\prime} k}\right\rangle, i^{\prime}=1,2, \ldots, p$, that is to say $\left\langle\mu_{i^{\prime} k}^{j}, v_{i^{\prime} k}^{j^{j}}\right\rangle^{i}$ are q-ROFVs in the brown part of Table 2. Assume that $i=0,1,2, \ldots, p$. A symbolization is given by

$$
\left\langle\mu_{i k}^{j}, v_{i k}^{j}\right\rangle= \begin{cases}\left\langle\mu_{j k}, v_{j k}\right\rangle, & \text { if } i=0 ; \\ \left\langle s_{i^{\prime} k}, t_{i^{\prime} k}\right\rangle, & \text { if } i>0 .\end{cases}
$$

On the above fundamental and crucial symbolic notion of the GGq-ROFSS, we contemplate novel averaging aggregation operators.

Definition 22. Consider Definition 14, where GGq-ROFSS $(\mathscr{Q}, \mathscr{A}, \eta)$ is given in Table 2 . Let $\Psi=\left[\varpi_{1}, \varpi_{2}, \ldots, \Phi_{n}\right]^{T}$ be the weighted vector over $\mathscr{A}$, such that $\sum_{k=1}^{n} \oplus_{k}=1$ and $\varpi_{k}>0$. Also take weighted vector $W=\left[\varphi_{0}, \varphi_{1}, \varphi_{2}, \ldots, \varphi_{p}\right]^{T}$ such that $\sum_{i=1}^{p} \omega_{i}=1$ and $\omega_{i}>0$, where $\varphi_{1}, \ldots, \varphi_{p}$ are weights for the judgements of the " $p$ " number of senior moderators and $\varphi_{0}$ is the weight for each q-ROFV in the light gray part of Table 2. In other words, $\varphi_{0}$ is the weight of whole data in 
Table 3: GGq-ROFSS $(\mathscr{Q}, \mathscr{A}, \eta)$ for $\mathscr{T}_{1}$.

\begin{tabular}{lcc}
\hline$\chi \mid \mathscr{A}$ & $e_{1}$ & $e_{2}$ \\
\hline$\rho_{1}$ & $\langle 0.8,0.8\rangle$ & $\langle 0.8,0.6\rangle$ \\
$\rho_{2}$ & $\langle 0.5,0.7\rangle$ & $\langle 0.4,0.3\rangle$ \\
$\rho_{3}$ & $\langle 0.6,0.8\rangle$ & $\langle 0.2,0.2\rangle$ \\
$\rho_{4}$ & $\langle 0.5,0.6\rangle$ & $\langle 0.6,0.1\rangle$ \\
$\rho_{5}$ & $\langle 0.7,0.3\rangle$ & $\langle 0.6,0.4\rangle$ \\
\hline$\alpha_{h_{1}}$ & $\langle 0.4,0.4\rangle$ & $\langle 0.7,0.7\rangle$ \\
$\alpha_{h_{2}}$ & $\langle 0.8,0.6\rangle$ & $\langle 0.6,0.6\rangle$ \\
\hline
\end{tabular}

TABle 4: GGq-ROFSS $\left(\mathscr{Q} \prime, \mathscr{B}, \eta^{\prime}\right)$ for $\mathscr{T}_{2}$.

\begin{tabular}{lccc}
\hline$\chi \mid \mathscr{B}$ & $e_{2}$ & $e_{4}$ & $e_{5}$ \\
\hline$\rho_{1}$ & $\langle 0.8,0.8\rangle$ & $\langle 0.8,0.6\rangle$ & $\langle 0.8,0.8\rangle$ \\
$\rho_{2}$ & $\langle 0.5,0.3\rangle$ & $\langle 0.5,0.7\rangle$ & $\langle 0.4,0.3\rangle$ \\
$\rho_{3}$ & $\langle 0.6,0.8\rangle$ & $\langle 0.5,0.5\rangle$ & $\langle 0.6,0.3\rangle$ \\
$\rho_{4}$ & $\langle 0.8,0.3\rangle$ & $\langle 0.5,0.6\rangle$ & $\langle 0.6,0.1\rangle$ \\
$\rho_{5}$ & $\langle 0.7,0.3\rangle$ & $\langle 0.6,0.5\rangle$ & $\langle 0.5,0.5\rangle$ \\
\hline$\beta_{h_{1}}^{\prime}$ & $\langle 0.4,0.4\rangle$ & $\langle 0.8,0.7\rangle$ & $\langle 0.7,0.7\rangle$ \\
$\beta_{h_{2}}^{\prime}$ & $\langle 0.8,0.2\rangle$ & $\langle 0.7,0.4\rangle$ & $\langle 0.6,0.6\rangle$ \\
\hline
\end{tabular}

q-ROFSS (see the light gray part of Table 2). Assume $i=0,1,2, \ldots, p$. Then, the generalized weighted q-Rung
TAble 5: Union of GGq-ROFSSs $(\mathscr{R}, \mathscr{A} \cup \mathscr{B}, \delta)$.

\begin{tabular}{lcccc}
\hline$\chi \mid \mathscr{A} \cup \mathscr{B}$ & $e_{1}$ & $e_{2}$ & $e_{4}$ & $e_{5}$ \\
\hline$\rho_{1}$ & $\langle 0.8,0.8\rangle$ & $\langle 0.8,0.8\rangle$ & $\langle 0.8,0.6\rangle$ & $\langle 0.8,0.8\rangle$ \\
$\rho_{2}$ & $\langle 0.5,0.7\rangle$ & $\langle 0.5,0.3\rangle$ & $\langle 0.5,0.3\rangle$ & $\langle 0.4,0.3\rangle$ \\
$\rho_{3}$ & $\langle 0.6,0.8\rangle$ & $\langle 0.6,0.8\rangle$ & $\langle 0.5,0.2\rangle$ & $\langle 0.6,0.3\rangle$ \\
$\rho_{4}$ & $\langle 0.5,0.6\rangle$ & $\langle 0.8,0.3\rangle$ & $\langle 0.6,0.1\rangle$ & $\langle 0.6,0.1\rangle$ \\
$\rho_{5}$ & $\langle 0.7,0.3\rangle$ & $\langle 0.7,0.3\rangle$ & $\langle 0.6,0.4\rangle$ & $\langle 0.5,0.5\rangle$ \\
\hline$\beta_{h_{1}}$ & $\langle 0.4,0.4\rangle$ & $\langle 0.4,0.4\rangle$ & $\langle 0.8,0.7\rangle$ & $\langle 0.7,0.7\rangle$ \\
$\beta_{h_{2}}$ & $\langle 0.8,0.6\rangle$ & $\langle 0.8,0.2\rangle$ & $\langle 0.7,0.4\rangle$ & $\langle 0.6,0.6\rangle$
\end{tabular}

TABLE 6: Intersection of GGq-ROFSSs $(\mathscr{R}, \mathscr{A} \cap \mathscr{B}, \delta)$.

\begin{tabular}{cc}
\hline$\chi \mid \mathscr{A} \cap \mathscr{B}$ & $e_{4}$ \\
\hline$\rho_{1}$ & $\langle 0.8,0.6\rangle$ \\
$\rho_{2}$ & $\langle 0.4,0.7\rangle$ \\
$\rho_{3}$ & $\langle 0.2,0.5\rangle$ \\
$\rho_{4}$ & $\langle 0.5,0.1\rangle$ \\
$\rho_{5}$ & $\langle 0.6,0.5\rangle$ \\
\hline$\beta_{h_{1}}$ & $\langle 0.7,0.7\rangle$ \\
$\beta_{h_{2}}$ & $\langle 0.6,0.6\rangle$ \\
\hline
\end{tabular}

orthopair fuzzy averaging operator (GWq-ROFA) undertaken by GGq-ROFSS is contemplated as follows:

$$
\begin{aligned}
& \Theta_{j}=\mathrm{GWq}-\operatorname{ROFA}\left(\left\langle\mu_{j 1}, v_{j 1}\right\rangle,\left\langle\mu_{j 2}, v_{j 2}\right\rangle, \ldots,\left\langle\mu_{j n}, v_{j n}\right\rangle\right) \\
& =\operatorname{q}-\operatorname{ROFWA}_{k}\left(\begin{array}{c}
\mathrm{q}-\operatorname{ROFWA}_{i}\left(\left\langle\mu_{01}^{j}, v_{01}^{j}\right\rangle,\left\langle\mu_{11}^{j}, v_{11}^{j}\right\rangle, \ldots,\left\langle\mu_{p 1}^{j}, v_{p 1}^{j}\right\rangle\right) \\
\mathrm{q}-\operatorname{ROFWA}_{i}\left(\left\langle\mu_{02}^{j}, v_{02}^{j}\right\rangle,\left\langle\mu_{12}^{j}, v_{12}^{j}\right\rangle, \ldots,\left\langle\mu_{p 2}^{j}, v_{p 2}^{j}\right\rangle\right) \\
\ldots \\
q-\operatorname{ROFWA}_{i}\left(\left\langle\mu_{0 n}^{j}, v_{0 n}^{j}\right\rangle,\left\langle\mu_{1 n}^{j}, v_{1 n}^{j}\right\rangle, \ldots,\left\langle\mu_{p n}^{j}, v_{p n}^{j}\right\rangle\right)
\end{array}\right) \\
& =\mathrm{q}-\operatorname{ROFWA}_{k}\left(\begin{array}{c}
\mathrm{q}-\operatorname{ROFWA}_{i}\left(\left\langle\mu_{j 1}, v_{j 1}\right\rangle,\left\langle s_{11}, t_{11}\right\rangle,\left\langle s_{21}, t_{21}\right\rangle, \ldots,\left\langle s_{p 1}^{j}, v_{p 1}^{j}\right\rangle\right) \\
\mathrm{q}-\operatorname{ROFWA}_{i}\left(\left\langle\mu_{j 2}, v_{j 2}\right\rangle,\left\langle s_{12}, t_{12}\right\rangle,\left\langle s_{22}, t_{22}\right\rangle, \ldots,\left\langle s_{p 2}, t_{p 2}\right\rangle\right) \\
\ldots \\
\mathrm{q}-\operatorname{ROFWA}_{i}\left(\left\langle\mu_{j n}, v_{j n}\right\rangle,\left\langle s_{1 n}, t_{1 n}\right\rangle,\left\langle s_{2 n}, t_{2 n}\right\rangle, \ldots,\left\langle s_{p n}, t_{p n}\right\rangle\right)
\end{array}\right),
\end{aligned}
$$

where $\mathrm{q}-\mathrm{ROFWA}_{k}$ is the q-rung orthopair fuzzy weighted averaging operator and it operates over the set of criteria, and $\mathrm{q}-\mathrm{ROFWA}_{i}$ is the q-rung orthopair fuzzy weighted averaging operator and it operates mutually over q-ROFSS and on the set of senior moderators.
The set of all GWq-ROFA operators for $m$ number of alternatives is indicated as $\Delta=\left\{\Theta_{1}, \Theta_{2}, \ldots, \Theta_{m}\right\}$. The above novel GWq-ROFA operators are realistic instruments for linear and entire aggregations of q-ROFVs in GGq-ROFSS. 
The GWq-ROFA operators have a specific way of incorporating each component of GGq-ROFSS. They entirely compel q-ROFVs in a linear way towards attributes until the final q-ROFV appears.
Example 2. Consider GGq-ROFSS indicated in Table 4 in Example 20. Given $\mathrm{q}-\mathrm{ROFV}\left(\rho_{1}\right)=(\langle 0.8,0.8\rangle,\langle 0.8,0.6\rangle$, $\langle 0.8,0.8\rangle)$. We have to calculate the GWq-ROFA operator for $\rho_{1}$. The q-ROFVs are depicted as

$$
\begin{aligned}
& \mathrm{q}-\operatorname{ROFWA}_{e_{2}}\left(\left\langle\mu_{11}, v_{11}\right\rangle,\left\langle s_{11}, t_{11}\right\rangle,\left\langle s_{21}, t_{21}\right\rangle\right)=(\langle 0.8,0.8\rangle,\langle 0.4,0.4\rangle,\langle 0.8,0.2\rangle), \\
& \mathrm{q}-\operatorname{ROFWA}_{e_{4}}\left(\left\langle\mu_{22}, v_{22}\right\rangle,\left\langle s_{12}, t_{12}\right\rangle,\left\langle s_{22}, t_{22}\right\rangle\right)=(\langle 0.8,0.6\rangle,\langle 0.8,0.7\rangle,\langle 0.7,0.4\rangle), \\
& \mathrm{q}-\operatorname{ROFWA}_{e_{5}}\left(\left\langle\mu_{23}, v_{23}\right\rangle,\left\langle s_{13}, t_{13}\right\rangle,\left\langle s_{23}, t_{23}\right\rangle\right)=(\langle 0.8,0.8\rangle,\langle 0.7,0.7\rangle,\langle 0.6,0.6\rangle) .
\end{aligned}
$$

Let $\Psi=\left[\Phi_{1}, \oplus_{2}, \oplus_{3}\right]^{T}=\left[\left(0.3 / e_{2}\right),\left(0.4 / e_{4}\right),\left(0.3 / e_{5}\right)\right]^{T}$ be the weighted vector over $\mathscr{A}$. Also take weighted vector $W=$ $\left[\varphi_{0}, \varphi_{1}, \varphi_{2}\right]^{T}=[0.40,0.25,0.35]^{T}$ for q-ROFSS and judgements of senior person/moderators. For $q=4$, we have

$$
\begin{aligned}
& q-\operatorname{ROFWA}(\langle 0.8,0.8\rangle,\langle 0.4,0.4\rangle,\langle 0.8,0.2\rangle)=\langle 0.7584,0.4141\rangle, \\
& q-\operatorname{ROFWA}(\langle 0.8,0.6\rangle,\langle 0.8,0.7\rangle,\langle 0.7,0.4\rangle)=\langle 0.7719,0.5411\rangle, \\
& q-\operatorname{ROFWA}(\langle 0.8,0.8\rangle,\langle 0.7,0.7\rangle,\langle 0.6,0.6\rangle)=\langle 0.7272,0.6996\rangle .
\end{aligned}
$$

Now, GWq-ROFA is given by $\Theta_{1}=\mathrm{GWq}-\mathrm{ROFA}$ $(\langle 0.8,0.8\rangle,\langle 0.8,0.6\rangle, \quad\langle 0.8,0.8\rangle)=\mathrm{q}-\operatorname{ROFWA}(\langle 0.7584$, $0.4141\rangle,\langle 0.7719,0.5411\rangle, \quad\langle 0.7272,0.6996\rangle)=\langle 0.7555$, $0.5394\rangle$. Similarly, GWq-ROFA operators $\Theta_{2}, \Theta_{3}, \Theta_{4}$, and $\Theta_{5}$ can be obtained.
Theorem 1. Let $a_{j k}=\left\langle\mu_{j} k, v_{j} k\right\rangle$ and $d_{i^{\prime} k}=\left\langle s_{i^{\prime} j}, t_{i^{\prime} j}\right\rangle$ be the $q$-ROFVs in GGq-ROFSS in Table 2, where $k=1,2, \ldots, n$, $j=1,2, \ldots, m$ and $i^{\prime}=1,2, \ldots, p$. If we consider $i=0,1,2$, ..., p, then the GWq-ROFA operator is given by

$$
\begin{aligned}
& \operatorname{GWq}-\operatorname{ROFA}\left(\left\langle\mu_{j 1}, v_{j 1}\right\rangle,\left\langle\mu_{j 2}, v_{j 2}\right\rangle, \ldots,\left\langle\mu_{j n}, v_{j n}\right\rangle\right) \\
&=\left\langle\sqrt[q]{\left.1-\prod_{k=1}^{n}\left(1-\left(1-\prod_{i=0}^{p}\left(1-\left(\mu_{i k}^{j}\right)^{q}\right)^{\varphi_{i}}\right)\right)^{\omega_{k}}, \prod_{k=1}^{n}\left(\prod_{i=0}^{p}\left(\left(v_{i k}^{j}\right)^{q}\right)^{\varphi_{i}}\right)^{\omega_{k}}\right\rangle .}\right.
\end{aligned}
$$

Proof. Assume that $p=1$ and $n=2$. Take $j=1$; we apply mathematical induction on $n$. By the definition of GGqROFSS,

$$
\begin{aligned}
\Theta_{j} & =\operatorname{GWq}-\operatorname{ROFWA}\left(\left\langle\mu_{11}, v_{11}\right\rangle,\left\langle\mu_{12}, v_{12}\right\rangle\right) \\
& =\operatorname{IFWA}_{j}\left(\begin{array}{l}
\operatorname{IWA}_{i}\left(\left\langle\mu_{01}^{1}, v_{01}^{1}\right\rangle,\left\langle\mu_{11}^{1}, v_{11}^{1}\right\rangle\right) \\
\operatorname{IWA}_{i}\left(\left\langle\mu_{02}^{1}, v_{02}^{1}\right\rangle,\left\langle\mu_{12}^{1}, v_{12}^{1}\right\rangle\right)
\end{array}\right)
\end{aligned}
$$

$$
=\operatorname{IFWA}_{j}\left(\begin{array}{c}
\operatorname{IWA}_{i}\left(\left\langle\mu_{11}, v_{11}\right\rangle,\left\langle s_{11}, t_{11}\right\rangle\right) \\
\operatorname{IWA}_{i}\left(\left\langle\mu_{12}, v_{12}\right\rangle,\left\langle s_{12}, t_{12}\right\rangle\right)
\end{array}\right) .
$$

Now, $\operatorname{IWA}_{i}\left(\left\langle\mu_{11}, \nu_{11}\right\rangle,\left\langle s_{11}, t_{11}\right\rangle\right)=$ $\left\langle\sqrt[q]{\left(1-\left(1-\left(\mu_{11}\right)^{q}\right)\right)^{\varphi_{0}} \cdot\left(1-\left(s_{11}\right)^{q}\right)^{\varphi_{1}}}, v_{11}^{\varphi_{0}} \cdot t_{11}^{\varphi_{1}}\right\rangle$, $\operatorname{IWA}_{i}\left(\left\langle\mu_{12}, \nu_{12}\right\rangle,\left\langle s_{12}, t_{12}\right\rangle\right)=$ $\left\langle\sqrt[q]{\left(1-\left(1-\left(\mu_{12}\right)^{q}\right)\right)^{\varphi_{0}} \cdot\left(1-\left(s_{12}\right)^{q}\right)^{\varphi_{1}}}, \nu_{12}^{\varphi_{0}} \cdot t_{12}^{\varphi_{1}}\right\rangle$. Thus, 


$$
\begin{aligned}
& \Theta_{j}=\mathrm{GWq}-\operatorname{ROFWA}\left(\left\langle\mu_{11}, \nu_{11}\right\rangle,\left\langle\mu_{12}, \nu_{12}\right\rangle\right) \\
& =\operatorname{IFWA}_{j}\left(\left\langle\sqrt[q]{1-\left(1-\left(\mu_{11}\right)^{q}\right)^{\varphi_{0}} \cdot\left(1-\left(s_{11}\right)^{q}\right)^{\varphi_{1}}}, v_{11}^{\varphi_{0}} \cdot t_{11}^{\varphi_{1}}\right\rangle,\left\langle\sqrt[q]{1-\left(1-\left(\mu_{12}\right)^{q}\right)^{\varphi_{0}} \cdot\left(1-\left(s_{12}\right)^{q}\right)^{\varphi_{1}}}, v_{12}^{\varphi_{0}} \cdot t_{12}^{\varphi_{1}}\right\rangle\right) \\
& =\left\langle\sqrt[q]{\left(1-\left(1-\left(1-\left(1-\left(\mu_{11}\right)^{q}\right)^{\varphi_{0}} \cdot\left(1-\left(s_{11}\right)^{q}\right)^{\varphi_{1}}\right)\right)^{\Phi_{1}}\right) \cdot\left(1-\left(1-\left(1-\left(1-\left(\mu_{12}\right)^{q}\right)^{\varphi_{0}} \cdot\left(1-\left(s_{12}\right)^{q}\right)^{\varphi_{1}}\right)\right)^{\Phi_{2}}\right)},\right. \\
& \left.\cdot\left(\nu_{11}^{\varphi_{0}} \cdot s_{11}^{\varphi_{1}}\right)^{\varpi_{1}} \cdot\left(\nu_{12}^{\varphi_{0}} \cdot s_{12}^{\varphi_{1}}\right)^{\varpi_{2}}\right\rangle \\
& =\left\langle\sqrt[q]{\left(1-\left(1-\left(1-\left(1-\left(\mu_{01}^{1}\right)^{q}\right)^{\varphi_{0}} \cdot\left(1-\left(\mu_{11}^{1}\right)^{q}\right)^{\varphi_{1}}\right)\right)^{\Phi_{1}}\right) \cdot\left(1-\left(1-\left(1-\left(1-\left(\mu_{02}^{1}\right)^{q}\right)^{\varphi_{0}} \cdot\left(1-\left(\mu_{12}^{1}\right)^{q}\right)^{\varphi_{1}}\right)\right)^{\Phi_{2}}\right)}\right. \\
& \left.\cdot\left(\left(v_{01}^{1}\right)^{\varphi_{0}} \cdot\left(v_{11}^{1}\right)^{\varphi_{1}}\right)^{\Phi_{1}} \cdot\left(\left(v_{02}^{1}\right)^{\varphi_{0}} \cdot\left(v_{12}^{1}\right)^{\varphi_{1}}\right)^{\Phi_{2}}\right\rangle \\
& =\left\langle\sqrt[q]{\left(1-\left(1-\left(1-\prod_{i=0}^{1}\left(1-\left(\mu_{i 1}^{1}\right)^{q}\right)^{\varphi_{i}}\right)\right)^{\Phi_{1}}\right) \cdot\left(1-\left(1-\left(1-\prod_{i=0}^{1}\left(1-\left(\mu_{i 2}^{1}\right)^{q}\right)^{\varphi_{i}}\right)\right)^{\Phi_{2}}\right)},\right. \\
& \left.\cdot\left(\prod_{i=0}^{1}\left(v_{i 1}^{1}\right)^{\varphi_{i}}\right)^{\Phi_{1}} \cdot\left(\prod_{i=0}^{1}\left(v_{i 2}^{1}\right)^{\varphi_{i}}\right)^{\Phi_{2}}\right\rangle \\
& =\left\langle\sqrt[q]{\left.1-\prod_{k=1}^{2}\left(1-\left(1-\prod_{i=0}^{1}\left(1-\left(\mu_{i k}^{1}\right)^{q}\right)^{\varphi_{i}}\right)\right)^{\Phi_{k}}, \prod_{k=1}^{2}\left(\prod_{i=0}^{1}\left(\left(v_{i k}^{1}\right)^{q}\right)^{\varphi_{i}}\right)^{\Phi_{k}}\right\rangle .}\right.
\end{aligned}
$$

Hence, the theorem is valid for $n=2$. Considering that this result is fine for $n=n^{\prime}$ that is

$$
\begin{aligned}
& \operatorname{GWq}-\operatorname{ROFA}\left(\left\langle\mu_{j 1}, v_{j 1}\right\rangle,\left\langle\mu_{j 2}, v_{j 2}\right\rangle, \ldots,\left\langle\mu_{j n^{\prime}}, v_{j n^{\prime}}\right\rangle\right) \\
&=\left\langle\sqrt[q]{\left.1-\prod_{k=1}^{n^{\prime}}\left(1-\left(1-\prod_{i=0}^{p}\left(1-\left(\mu_{i k}^{j}\right)^{q}\right)^{\varphi_{i}}\right)\right)^{\Phi_{k}}, \prod_{k=1}^{n^{\prime}}\left(\prod_{i=0}^{p}\left(\left(v_{i k}^{j}\right)^{q}\right)^{\varphi_{i}}\right)^{\Phi_{k}}\right\rangle .}\right.
\end{aligned}
$$

Then, for $n=n^{\prime}+1$, we have

$$
\begin{aligned}
& \operatorname{GWq}-\operatorname{ROFA}\left(\left\langle\mu_{j 1}, v_{j 1}\right\rangle,\left\langle\mu_{j 2}, v_{j 2}\right\rangle, \ldots,\left\langle\mu_{j\left(n^{\prime}+1\right)}, v_{j\left(n^{\prime}+1\right)}\right\rangle\right) \\
& =\left\langle\sqrt{q} 1-\prod_{k=1}^{n^{\prime}+1}\left(1-\left(1-\prod_{i=0}^{p}\left(1-\left(\mu_{i k}^{j}\right)^{q}\right)^{\varphi_{i}}\right)\right)^{\omega_{k}}, \prod_{k=1}^{n^{\prime}+1}\left(\prod_{i=0}^{p}\left(\left(v_{i k}^{j}\right)^{q}\right)^{\varphi_{i}}\right)^{\omega_{k}}\right\rangle .
\end{aligned}
$$

Hence, by mathematical induction, Theorem 1 satisfy for all positive integer $n$.

Theorem 2. (idempotency) If $\left\langle\mu_{j k}, v_{j k}\right\rangle=\left\langle\mu_{j}, v_{j}\right\rangle$ and $\left\langle\mu_{i^{\prime} k}, v_{i^{\prime} k}\right\rangle=\langle\mu, v\rangle=\left\langle\mu_{j}, v_{j}\right\rangle$ for all $k=1,2, \ldots, n$, then
$G W q-R O F A\left(\left\langle\mu_{j 1}, v_{j 1}\right\rangle,\left\langle\mu_{j 2}, v_{j 2}\right\rangle\right.$, $\left.\ldots,\left\langle\mu_{j n}, v_{j n}\right\rangle\right)=\langle\mu, v\rangle$.

Proof. Given $\left\langle\mu_{j k}, v_{j k}\right\rangle=\langle\mu, v\rangle$ and $\left\langle\mu_{i^{\prime} k}, \nu_{i^{\prime} k}\right\rangle=\langle\mu, v\rangle$ for all $i^{\prime}=1,2, \ldots, p$ and $k=1,2, \ldots, n$.

$$
\Theta_{j}=\mathrm{GWq}-\operatorname{ROFA}\left(\left\langle\mu_{j 1}, v_{j 1}\right\rangle,\left\langle\mu_{j 2}, v_{j 2}\right\rangle, \ldots,\left\langle\mu_{j n}, v_{j n}\right\rangle\right)
$$




$$
\begin{aligned}
& =\left\langle\sqrt[q]{1-\prod_{k=1}^{n}\left(1-\left(1-\prod_{i=0}^{p}\left(1-\left(\mu_{i k}^{j}\right)^{q}\right)^{\varphi_{i}}\right)\right)^{\Phi_{k}}}, \prod_{k=1}^{n}\left(\prod_{i=0}^{p}\left(\left(v_{i k}^{j}\right)^{q}\right)^{\varphi_{i}}\right)^{\Phi_{k}}\right\rangle \\
& =\left\langle\sqrt[q]{1-\prod_{k=1}^{n}\left(1-\left(1-\left(1-(\mu)^{q}\right) \sum_{i=0}^{p} \varphi_{i}\right)\right)^{\Phi_{k}}}, \prod_{k=1}^{n}\left(\left((\nu)^{q}\right)^{\sum_{i=0}^{p} \varphi_{i}}\right)^{\Phi_{k}}\right\rangle \\
& =\left\langle\sqrt[q]{1-\prod_{k=1}^{n}\left(1-\left(1-\left(1-(\mu)^{q}\right)\right)\right)^{\Phi_{k}}}, \prod_{k=1}^{n}\left(\left((\nu)^{q}\right)\right)^{\Phi_{k}}\right\rangle \\
& =\left\langle\sqrt[q]{1-\left(1-\left(1-\left(1-(\mu)^{q}\right)\right)\right) \sum_{k=1}^{n} \oplus_{k}},\left(\left((\nu)^{q}\right)\right) \sum_{k=1}^{n} \varpi_{k}\right\rangle=\langle\mu, \nu\rangle, \\
& \operatorname{GWq}-\operatorname{ROFA}\left(\left\langle\mu_{j 1}, v_{j 1}\right\rangle,\left\langle\mu_{j 2}, v_{j 2}\right\rangle, \ldots,\left\langle\mu_{j n}, v_{j n}\right\rangle\right)=\langle\mu, v\rangle .
\end{aligned}
$$

Theorem 3. (boundedness) If $\left\langle\mu^{+}, v^{-}\right\rangle=\left\langle\left(\mu_{i k}^{j}\right)^{\max }\right.$, $\left.\left(v_{i k}^{j}\right)^{\min }\right\rangle$ and $\left\langle\mu^{-}, \nu^{+}\right\rangle=\left\langle\left(\mu_{i k}^{j}\right)^{\min },\left(\nu_{i k}^{j}\right)^{\max }\right\rangle$ for all $j=1,2$, $\ldots, m$ and $i=0,1,2, \ldots, p$, then $\left\langle\mu^{-}, \nu^{+}\right\rangle \leq G W q-R O F A$ $\left(\left\langle\mu_{j 1}, v_{j 1}\right\rangle,\left\langle\mu_{j 2}, v_{j 2}\right\rangle, \ldots,\left\langle\mu_{j n}, v_{j n}\right\rangle\right) \leq\left\langle\mu^{+}, v^{-}\right\rangle$.

Proof. Since $\mu^{-} \leq \mu_{i k}^{j} \leq \mu^{+} \Leftrightarrow\left(\mu^{-}\right)^{q} \leq\left(\mu_{i k}^{j}\right)^{q} \leq \quad\left(\mu^{+}\right)^{q} \Leftrightarrow$ $1-\left(\mu^{-}\right)^{q} \geq 1-\left(\mu_{i k}^{j}\right)^{q} \geq 1-\left(\mu^{+}\right)^{q} \Leftrightarrow \prod_{i=0}^{p}\left(1-\left(\mu^{-}\right)^{q}\right)^{\varphi_{i}} \geq$ $\prod_{i=0}^{p}\left(1-\left(\mu_{i k}^{j}\right)^{q}\right)^{\varphi_{i}} \geq \prod_{i=0}^{p}\left(1-\left(\mu^{+}\right)^{q}\right)^{\varphi_{i}} \Leftrightarrow\left(1-\left(\mu^{-}\right)^{q}\right) \sum_{i=0}^{p} \varphi_{i}$ $\geq \prod_{i=0}^{p}\left(1-\left(\mu_{i k}^{j}\right)^{q}\right)^{\varphi_{i}} \geq\left(1-\left(\mu^{+}\right)^{q}\right) \sum_{i=0}^{p} \varphi_{i} \Leftrightarrow\left(1-\left(\mu^{-}\right)^{q}\right) \geq$ $\prod_{i=0}^{p}\left(1-\left(\mu_{i k}^{j}\right)^{q}\right)^{\varphi_{i}} \geq\left(1-\left(\mu^{+}\right)^{q}\right) \Leftrightarrow 1-\left(1-\left(\mu^{-}\right)^{q}\right) \leq 1-\prod_{i=0}^{p}$ $\left(1-\left(\mu_{i k}^{j}\right)^{q}\right)^{\varphi_{i}} \leq 1-\left(1-\left(\mu^{+}\right)^{q}\right) \Leftrightarrow\left(\mu^{-}\right)^{q} \leq 1-\prod_{i=0}^{p}(1-$ $\left.\left(\mu_{i k}^{j}\right)^{q}\right)^{\varphi_{i}} \leq\left(\mu^{+}\right)^{q} \Leftrightarrow 1-\left(\mu^{-}\right)^{q} \geq 1-\left(1-\prod_{i=0}^{p}\left(1-\left(\mu_{i k}^{j}\right)^{q}\right)^{\varphi_{i}}\right)$ $\geq 1-\left(\mu^{+}\right)^{q} \Leftrightarrow \prod_{k=1}^{n}\left(1-\left(\mu^{-}\right)^{q}\right)^{\Phi_{k}} \geq \prod_{k=1}^{n}\left(1-\left(1-\prod_{i=0}^{p}\right.\right.$ $\left.\left.\left(1-\left(\mu_{i k}^{j}\right)^{q}\right)^{\varphi_{i}}\right)\right)^{\Phi_{k}} \geq \quad \prod_{k=1}^{n}\left(1-\left(\mu^{+}\right)^{q}\right)^{\Phi_{k}} \Leftrightarrow\left(1-\left(\mu^{-}\right)^{q}\right)$ $\sum_{k=1}^{n} \omega_{k} \geq \prod_{k=1}^{n}\left(1-\left(1-\prod_{i=0}^{p} \quad\left(1-\left(\mu_{i k}^{j}\right)^{q}\right)^{\varphi_{i}}\right)\right) \geq\left(1-\left(\mu^{+}\right)^{q}\right)$ $\sum_{k=1}^{n} \Phi_{k} \Leftrightarrow\left(1-\left(\mu^{-}\right)^{q}\right) \geq \prod_{k=1}^{n} \quad\left(1-\left(1-\prod_{i=0}^{p}\left(1-\left(\mu_{i k}^{j}\right)^{q}\right)^{\varphi_{i}}\right)\right)$ $\varpi_{k} \geq\left(1-\left(\mu^{+}\right)^{q}\right) \Leftrightarrow 1-\left(1-\left(\mu^{-}\right)^{q}\right) \leq 1-\prod_{k=1}^{n}\left(1-\left(1-\prod_{i=0}^{p}\right.\right.$ $\left.\left.\left(1-\left(\mu_{i k}^{j}\right)^{q}\right) \varphi_{i}\right)\right)^{\Phi_{k}} \leq 1-\left(1-\left(\mu^{+}\right)^{q}\right) \Leftrightarrow\left(\mu^{-}\right)^{q} \leq 1-\prod_{k=1}^{n}(1-$ $\left.\left(1-\quad \prod_{i=0}^{p}\left(1-\left(\mu_{i k}^{j}\right)^{q}\right)^{\varphi_{i}}\right)\right)^{\Phi_{k}} \leq\left(\mu^{+}\right)^{q} \quad \Leftrightarrow\left(\mu^{-}\right) \quad \leq$ $\sqrt[q]{1-\prod_{k=1}^{n}\left(1-\left(1-\prod_{i=0}^{p}\left(1-\left(\mu_{i k}^{j}\right)^{q}\right)^{\varphi_{i}}\right)\right)^{\Phi_{k}}} \leq\left(\mu^{+}\right)$.

Similarly, nonmembership part is aggregated as $\left(\mu^{+}\right) \leq \prod_{k=1}^{n}\left(\prod_{i=0}^{p}\left(\left(v_{i k}^{j}\right)^{q}\right)^{\varphi_{i}}\right)^{\Phi_{k}} \leq\left(\mu^{-}\right)$. This concluded the proof of the theorem, $\left\langle\mu^{-}, \nu^{+}\right\rangle \leq \mathrm{GWq}-$ $\operatorname{ROFA}\left(\left\langle\mu_{j 1}, v_{j 1}\right\rangle,\left\langle\mu_{j 2}, v_{j 2}\right\rangle, \ldots,\left\langle\mu_{j n}, v_{j n}\right\rangle\right) \leq\left\langle\mu^{+}, v^{-}\right\rangle$.

Theorem 4. (monotonicity) If $\left\langle\mu_{i k}^{j}, v_{i k}^{j}\right\rangle$ and $\left\langle\bar{\mu}_{i k}^{j}, \bar{v}_{i k}^{j}\right\rangle$ for all $i=\{0,1,2, \ldots, p\}, j=\{1,2, \ldots, m\}, k=\{1,2, \ldots, n\}$ are two IFVs such that $\left\langle\mu_{i k}^{j}, v_{i k}^{j}\right\rangle \leq\left\langle\bar{\mu}_{i k}^{j}, \bar{v}_{i k}^{j}\right\rangle$, then $G W q-R O F A$ $\left(\left\langle\mu_{j 1}, v_{j 1}\right\rangle,\left\langle\mu_{j 2}, v_{j 2}\right\rangle, \ldots,\left\langle\mu_{j n}, v_{j n}\right\rangle\right) \leq G W q-\operatorname{ROFA}\left(\left\langle\bar{\mu}_{j 1}\right.\right.$, $\left.\left.\bar{\nu}_{j 1}\right\rangle,\left\langle\bar{\mu}_{j 2}, \bar{v}_{j 2}\right\rangle, \ldots,\left\langle\bar{\mu}_{j n}, \bar{v}_{j n}\right\rangle\right)$.

Proof. It can be concluded from Theorem 3.

Proposition 2. Let $(\mathcal{Q}, \mathscr{A}, \eta)$ be a $G G q-R O F S S$, given in Table 2. Then,

(1) If $\left\langle s_{i^{\prime} k}, t_{i^{\prime} k}\right\rangle=\langle 1,0\rangle$ for all $i^{\prime}$ and $k$, then $\Theta_{j}=\{\langle 1,0\rangle,\langle 1,0\rangle, \ldots,\langle 1,0\rangle\}$.

(2) If $\left\langle\mu_{0 k}^{j}, v_{0 k}^{j}\right\rangle=\left\langle\mu_{j k}, v_{j k}\right\rangle=\langle 1,0\rangle$ for all $j$ and $k$, then $\Theta_{j}=\{\langle 1,0\rangle,\langle 1,0\rangle, \ldots,\langle 1,0\rangle\}$.

(3) If $\left\langle s_{i^{\prime} k}, t_{i^{\prime} k}\right\rangle=\langle 1,0\rangle$ for all $i^{\prime}, k$ and $\left\langle\mu_{0 k}^{j}, v_{0 k}^{j}\right\rangle$ $=\left\langle\mu_{j k}, v_{j k}\right\rangle=\langle 1,0\rangle$ for all $j, k$, then $\Theta_{j}=\{\langle 1,0\rangle,\langle 1,0\rangle, \ldots,\langle 1,0\rangle\}$.

(4) If $\left\langle s_{i^{\prime} k}, t_{i^{\prime} k}\right\rangle=\langle 0,1\rangle$ for all $i^{\prime}, k$ and $\left\langle\mu_{0 k}^{j}, v_{0 k}^{j}\right\rangle$ $=\left\langle\mu_{j k}, v_{j k}\right\rangle=\langle 0,1\rangle$ for all $j, k$ then $\Theta_{j}=\{\langle 0,1\rangle$, $\langle 0,1\rangle, \ldots,\langle 0,1\rangle\}$.

Definition 23. Consider Definition 14, where GGq-ROFSS $(\mathscr{Q}, \mathscr{A}, \eta)$ is given in Table 2 . Let $\Psi=\left[\varpi_{1}, \varpi_{2}, \ldots, \varpi_{n}\right]^{T}$ be the weighted vector over $\mathscr{A}$, such that $\sum_{k=1}^{n} \varpi_{k}=1$ and $\omega_{k}>0$. Also take weighted vector $W=\left[\varphi_{0}, \varphi_{1}, \varphi_{2}, \ldots, \varphi_{p}\right]^{T}$ such that $\sum_{i=1}^{p} \omega_{i}=1$ and $\omega_{i}>0$, where $\varphi_{1}, \ldots, \varphi_{p}$ are weights for the judgements of the " $p$ " number of senior moderators and $\varphi_{0}$ is the weight for each q-ROFV in the light gray part of Table 2. In other words, $\varphi_{0}$ is the weight of whole data in q-ROFSS (see the light gray part of Table 2). Assume $i=0,1,2, \ldots, p$. Then, the generalized weighted q-Rung orthopair fuzzy geometric operator (GWq-ROFG) undertook by GGq-ROFSS is contemplated as follows:

$$
\Theta_{j}=\mathrm{GWq}-\operatorname{ROFG}\left(\left\langle\mu_{j 1}, v_{j 1}\right\rangle,\left\langle\mu_{j 2}, v_{j 2}\right\rangle, \ldots,\left\langle\mu_{j n}, v_{j n}\right\rangle\right)
$$




$$
\left.\begin{array}{c}
=\mathrm{q}-\operatorname{ROFWG}_{k}\left(\begin{array}{c}
\mathrm{q}-\operatorname{ROFWG}_{i}\left(\left\langle\mu_{01}^{j}, v_{01}^{j}\right\rangle,\left\langle\mu_{11}^{j}, v_{11}^{j}\right\rangle, \ldots,\left\langle\mu_{p 1}^{j}, v_{p 1}^{j}\right\rangle\right) \\
\mathrm{q}-\operatorname{ROFWG}_{i}\left(\left\langle\mu_{02}^{j}, v_{02}^{j}\right\rangle,\left\langle\mu_{12}^{j}, v_{12}^{j}\right\rangle, \ldots,\left\langle\mu_{p 2}^{j}, v_{p 2}^{j}\right\rangle\right) \\
\ldots \\
\mathrm{q}-\operatorname{ROFWG}_{i}\left(\left\langle\mu_{0 n}^{j}, v_{0 n}^{j}\right\rangle,\left\langle\mu_{1 n}^{j}, v_{1 n}^{j}\right\rangle, \ldots,\left\langle\mu_{p n}^{j}, v_{p n}^{j}\right\rangle\right)
\end{array}\right) \\
=\mathrm{q}_{-} \operatorname{ROFWG}_{k}\left(\begin{array}{c}
\mathrm{q}-\operatorname{ROFWG}_{i}\left(\left(\mu_{j 1}, v_{j 1}\right),\left\langle s_{11}, t_{11}\right\rangle,\left\langle s_{21}, t_{21}\right\rangle, \ldots,\left\langle s_{p 1}^{j}, v_{p 1}^{j}\right\rangle\right) \\
\mathrm{q}-\operatorname{ROFWG}_{i}\left(\left\langle\mu_{j 2}, v_{j 2}\right\rangle,\left\langle s_{12}, t_{12}\right\rangle,\left\langle s_{22}, t_{22}\right\rangle, \ldots,\left\langle s_{p 2}, t_{p 2}\right\rangle\right) \\
\mathrm{q}-\operatorname{ROFWG}_{i}\left(\left\langle\mu_{j n}, v_{j n}\right\rangle,\left\langle s_{1 n}, t_{1 n}\right\rangle,\left\langle s_{2 n}, t_{2 n}\right\rangle, \ldots,\left\langle s_{p n}, t_{p n}\right\rangle\right.
\end{array}\right),
\end{array}\right),
$$

where $\mathrm{q}-\mathrm{ROFWG}_{k}$ is the q-rung orthopair fuzzy weighted geometric operator and it operates over the set of criteria, and $\mathrm{q}-\mathrm{ROFWG}_{i}$ is the q-rung orthopair fuzzy weighted geometric operator and it operates mutually over q-ROFSS and on the set of senior moderators.

The set of all GWq-ROFG operators for $m$ number of alternatives is indicated as $\Delta \prime=\left\{\Theta_{1}^{\prime}, \Theta_{2}^{\prime}, \ldots, \Theta_{m}^{\prime}\right\}$. The above novel GWq-ROFG operators are realistic instruments for linear and entire aggregations of q-ROFVs in GGq-ROFSS.
The GWq-ROFG operators have a specific way of incorporating each component of GGq-ROFSS. They entirely compel q-ROFVs in a linear way towards attributes until the final q-ROFV appears.

Example 3. Consider GGq-ROFSS indicated in Table 4 in Example 3. Given $\mathrm{q}-\operatorname{ROFV}\left(\rho_{2}\right)=(\langle 0.5,0.3\rangle,\langle 0.5,0.7\rangle$, $\langle 0.4,0.3\rangle)$, we have to calculate the GWq-ROFG operator for $\rho_{1}$. The q-ROFVs are depicted as

$$
\begin{aligned}
& \mathrm{q}-\operatorname{ROFWG}_{e_{2}}\left(\left\langle\mu_{21}, v_{21}\right\rangle,\left\langle s_{11}, t_{11}\right\rangle,\left\langle s_{21}, t_{21}\right\rangle\right)=(\langle 0.5,0.3\rangle,\langle 0.4,0.4\rangle,\langle 0.8,0.2\rangle), \\
& \mathrm{q}-\operatorname{ROFWG}_{e_{4}}\left(\left\langle\mu_{22}, v_{22}\right\rangle,\left\langle s_{12}, t_{12}\right\rangle,\left\langle s_{22}, t_{22}\right\rangle\right)=(\langle 0.5,0.7\rangle,\langle 0.8,0.7\rangle,\langle 0.7,0.4\rangle), \\
& \mathrm{q}-\operatorname{ROFWG}_{e_{5}}\left(\left\langle\mu_{23}, v_{23}\right\rangle,\left\langle s_{13}, t_{13}\right\rangle,\left\langle s_{23}, t_{23}\right\rangle\right)=(\langle 0.4,0.3\rangle,\langle 0.7,0.7\rangle,\langle 0.6,0.6\rangle) .
\end{aligned}
$$

Let $\Psi=\left[\varpi_{1}, \oplus_{2}, \oplus_{3}\right]^{T}=\left[\left(0.3 / e_{2}\right),\left(0.4 / e_{4}\right),\left(0.3 / e_{5}\right)\right]^{T}$ be the weighted vector over $\mathscr{A}$. Also take weighted vector $W=$ $\left[\varphi_{0}, \varphi_{1}, \varphi_{2}\right]^{T}=[0.40,0.25,0.35]^{T}$ for q-ROFSS and judgements of senior person/moderators. For $q=4$, we have

$$
\begin{aligned}
& q-\operatorname{ROFWG}(\langle 0.5,0.3\rangle,\langle 0.4,0.4\rangle,\langle 0.8,0.2\rangle)=\langle 0.6644,0.2797\rangle, \\
& q-\operatorname{ROFWG}(\langle 0.5,0.7\rangle,\langle 0.8,0.7\rangle,\langle 0.7,0.4\rangle)=\langle 0.6880,0.5755\rangle, \\
& q-\operatorname{ROFWG}(\langle 0.4,0.3\rangle,\langle 0.7,0.7\rangle,\langle 0.6,0.6\rangle)=\langle 0.5883,0.4726\rangle .
\end{aligned}
$$

Now GWq-ROFG is given by

$$
\begin{aligned}
\Theta_{2}^{\prime} & =\mathrm{GWq}-\operatorname{ROFG}(\langle 0.5,0.3\rangle,\langle 0.5,0.7\rangle,\langle 0.4,0.3\rangle) \\
& =\mathrm{q}-\operatorname{ROFWG}(\langle 0.6644,0.2797\rangle,\langle 0.6880,0.5755\rangle,\langle 0.5883,0.4726\rangle) \\
& =\langle 0.6560,0.4369\rangle .
\end{aligned}
$$

Similarly, GWq-ROFG operators $\Theta_{1}^{\prime}, \Theta_{3}^{\prime}, \Theta_{4}^{\prime}$, and $\Theta_{5}^{\prime}$ can be obtained.
Theorem 5. Let $a_{j k}=\left\langle\mu_{j} k, v_{j} k\right\rangle$ and $d_{i^{\prime} k}=\left\langle s_{i^{\prime} j}, t_{i^{\prime} j}\right\rangle$ be the $q$-ROFVs in GGq-ROFSS in Table 2, where $k=1,2, \ldots, n$, 
$j=1,2, \ldots, m$, and $i^{\prime}=1,2, \ldots, p$. If we consider $i=0,1,2, \ldots, p$, then $G W q-R O F G$ operator is given by

$$
\begin{aligned}
& \operatorname{GWq}-\operatorname{ROFG}\left(\left\langle\mu_{j 1}, v_{j 1}\right\rangle,\left\langle\mu_{j 2}, v_{j 2}\right\rangle, \ldots,\left\langle\mu_{j n}, v_{j n}\right\rangle\right) \\
& =\left\langle\prod_{k=1}^{n}\left(\prod_{i=0}^{p}\left(\left(\mu_{i k}^{j}\right)^{q}\right)^{\varphi_{i}}\right)^{\Phi_{k}}, \sqrt{[q]} 1-\prod_{k=1}^{n}\left(1-\left(1-\prod_{i=0}^{p}\left(1-\left(v_{i k}^{j}\right)^{q}\right)^{\varphi_{i}}\right)\right)^{\omega_{k}}\right\rangle .
\end{aligned}
$$

Proof. Same as the proof of Theorem 1.

Theorem 6. (idempotency) If $\left\langle\mu_{j k}, v_{j k}\right\rangle=\left\langle\mu_{j}, v_{j}\right\rangle$ and $\left\langle\mu_{i^{\prime} k}, v_{i^{\prime} k}\right\rangle=\langle\mu, v\rangle=\left\langle\mu_{j}, v_{j}\right\rangle$ for all $k=1,2, \ldots, n$, then $G W q-\operatorname{ROFG}\left(\left\langle\mu_{j 1}, v_{j 1}\right\rangle,\left\langle\mu_{j 2}, v_{j 2}\right\rangle, \quad \ldots,\left\langle\mu_{j n}, v_{j n}\right\rangle\right)=$ $\langle\mu, v\rangle$.

Proof. Same as the proof of Theorem 2.

Theorem 7. (boundedness) If $\left\langle\mu^{+}, \nu^{-}\right\rangle=\left\langle\left(\mu_{i k}^{j}\right)^{\max }\right.$, $\left.\left(\nu_{i k}^{j}\right)^{\min }\right\rangle$ and $\left\langle\mu^{-}, \nu^{+}\right\rangle=\left\langle\left(\mu_{i k}^{j}\right)^{\min },\left(\nu_{i k}^{j}\right)^{\max }\right\rangle$ for all $j=$ $1,2, \ldots, m$ and $i=0,1,2, \ldots, p$, then $\left\langle\mu^{-}, \nu^{+}\right\rangle \leq G W q$ $-\operatorname{ROFG}\left(\left\langle\mu_{j 1}, v_{j 1}\right\rangle,\left\langle\mu_{j 2}, v_{j 2}\right\rangle, \ldots,\left\langle\mu_{j n}, v_{j n}\right\rangle\right) \leq\left\langle\mu^{+}, v^{-}\right\rangle$.

Proof. Same as the proof of Theorem 3.

Theorem 8. (monotonicity) If $\left\langle\mu_{i k}^{j}, v_{i k}^{j}\right\rangle$ and $\left\langle\bar{\mu}_{i k}^{j}, \bar{v}_{i k}^{j}\right\rangle$ for all $i=\{0,1,2, \ldots, p\}, j=\{1,2, \ldots, m\}, k=\{1,2, \ldots, n\}$ are two IFVs such that $\left\langle\mu_{i k}^{j}, v_{i k}^{j}\right\rangle \leq\left\langle\bar{\mu}_{i k}^{j}, \bar{v}_{i k}^{j}\right\rangle$, then

$$
\begin{aligned}
& \operatorname{GWq}-\operatorname{ROFG}\left(\left\langle\mu_{j 1}, v_{j 1}\right\rangle,\left\langle\mu_{j 2}, v_{j 2}\right\rangle, \ldots,\left\langle\mu_{j n}, v_{j n}\right\rangle\right) \\
& \quad \leq \operatorname{GWq}-\operatorname{ROFG}\left(\left\langle\bar{\mu}_{j 1}, \bar{v}_{j 1}\right\rangle,\left\langle\bar{\mu}_{j 2}, \bar{v}_{j 2}\right\rangle, \ldots,\left\langle\bar{\mu}_{j n}, \bar{v}_{j n}\right\rangle\right) .
\end{aligned}
$$

Proof. It can be concluded from Theorem 4.

Proposition 3. Let $(\mathcal{Q}, \mathscr{A}, \eta)$ be a GGq-ROFSS, given in Table 2. Then,

(1) If $\left\langle s_{i^{\prime} k}, t_{i^{\prime} k}\right\rangle=\langle 0,1\rangle$ for all $i^{\prime}$ and $k$, then $\Theta_{j}^{\prime}=\{\langle 0,1\rangle$, $\langle 0,1\rangle, \ldots,\langle 0,1\rangle\}$.

(2) If $\left\langle\mu_{0 k}^{j}, v_{0 k}^{j}\right\rangle=\left\langle\mu_{j k}, v_{j k}\right\rangle=\langle 0,1\rangle$ for all $j$ and $k$, then $\Theta_{j}^{\prime}=\{\langle 0,1\rangle,\langle 0,1\rangle, \ldots,\langle 0,1\rangle\}$.

(3) If $\left\langle s_{i^{\prime} k}, t_{i^{\prime} k}\right\rangle=\langle 0,1\rangle$ for all $i^{\prime}, k$ and $\left\langle\mu_{0 k}^{j}, v_{0 k}^{j}\right\rangle=$ $\left\langle\mu_{j k}, v_{j k}\right\rangle=\langle 0,1\rangle$ for all $j, k$ then $\Theta_{j}^{\prime}=\{\langle 0,1\rangle$, $\langle 0,1\rangle, \ldots,\langle 0,1\rangle\}$.

(4) If $\left\langle s_{i^{\prime} k}, t_{i^{\prime} k}\right\rangle=\langle 1,0\rangle$ for all $i^{\prime}, k$ and $\left\langle\mu_{0 k}^{j}, v_{0 k}^{j}\right\rangle$ $=\left\langle\mu_{j k}, v_{j k}\right\rangle=\langle 1,0\rangle$ for all $j, k$ then $\Theta_{j}^{\prime}=\{\langle 1,0\rangle$, $\langle 1,0\rangle, \ldots,\langle 1,0\rangle\}$.

\section{Multicriteria Decision-Making Method}

In this section, a methodology on proposed operators is introduced, and for application, a numerical application is investigated.
6.1. Methodology. Let $\mathscr{X}=\left\{\rho_{1}, \rho_{2}, \ldots, \rho_{m}\right\}$ be the set of alternatives for a problem in which we have to choose the best alternative. Every alternative possesses the specific q-ROFVs of a set of criteria/attributes $E$. Let $\mathscr{A} \subseteq E$. An expert's committee give the q-ROFVs on set $\mathscr{X}$ comprising attributes in the form of q-ROFSS. The final examination of the q-ROFSS is provided by some senior experts/moderators in the form of $\mathrm{q}-\mathrm{ROF}$ over $\mathscr{A}=\left\{e_{1}, e_{2}, \ldots, e_{n}\right\}$. This forms GGq-ROFSS $(\mathscr{Q}, \mathscr{A}, \eta)$ given in Table 2 in Definition 14 . In real-life problem, usually, the importance of attributes is given in the form of a weighted vector. Let $\Psi=\left[\varphi_{1}, \varphi_{2}, \ldots\right.$, $\left.\varphi_{n}\right]^{T}$ be the weighted vector over $\mathscr{A}$, such that $\sum_{k=1}^{n} \varphi_{k}=1$, $\varphi_{k}>0$. Also take weighted vector $W=\left[\varpi_{0}, \varpi_{1}, \varpi_{2}, \ldots, \varpi_{p}\right]^{T}$ such that $\sum_{i^{\prime}=0}^{p} \Phi_{i}=1, \omega_{i}>0$ where $\omega_{1}, \oplus_{2}, \ldots, \oplus_{p}$ are the weights for the judgements of the " $p$ " number of senior moderators, and $\omega_{0}$ is the weight for each q-ROFV in the light gray part of Table 2 . In other words, $\omega_{0}$ is the weight of whole data in q-ROFSS (see the light gray part of Table 2). Next, the normalization of the q-ROFVs in $(\mathcal{Q}, \mathscr{A}, \eta) \forall i, j, k$ is obtained by using the following equation:

$$
\left\langle\mu_{i k}^{j}, v_{i k}^{j}\right\rangle= \begin{cases}\left\langle\mu_{i k}^{j}(e), v_{i k}^{j}(e)\right\rangle, & \text { if } e \text { is benefit kind criteria, } \\ \left\langle v_{i k}^{j}(e), \mu_{i k}^{j}(e)\right\rangle, & \text { if } e \text { is cost kind criteria. }\end{cases}
$$

This designs a new GGq-ROFSS $\left(\mathcal{Q} \prime, \mathscr{A}, \eta^{\prime}\right)$. Then, we compute GWq-ROFA or GWq-ROFG operators on $(\mathcal{Q} \prime, \mathscr{A}$, $\eta \prime)$ and calculate score function on each GWq-ROFA or GWq-ROFG. Finally, rank the alternatives on the real values of score functions.

In order to evaluate most suitable alternative, we numerate the steps of the MCDM method in an algorithm as follows.

This algorithm is shown in Figure 1.

6.2. Numerical Example. An automation company wants to choose a director for their Research and Development ( $R$ and D) department from four applicants $\rho_{1}, \rho_{2}, \rho_{3}$, and $\rho_{4}$. The crucial attributes for evaluation are

$$
\begin{aligned}
& C_{1}: \text { personality } \\
& C_{2}: \text { self-confidence } \\
& C_{3}: \text { score obtained in a college degree } \\
& C_{4}: \text { administrative skills } \\
& C_{5}: \text { experience } \\
& C_{6}: \text { abilities in research areas }
\end{aligned}
$$


(1) Consider a set of alternatives say $\mathscr{X}=\left\{\rho_{1}, \rho_{2}, \ldots, \rho_{s}\right\}$ their attributes as a set $E$. $\mathscr{A}=\left\{e_{1}, e_{2}, \ldots, e_{n}\right\}$.

(2) Constitute $r$ number of committees of experts $C M_{1}, C M_{2}, \ldots, C M_{r}$ comprising sets of attributes $\mathscr{A}_{1}, \mathscr{A}_{2}, \ldots, \mathscr{A}_{r}$ respectively, where $\mathscr{A}_{1} \subset E, \mathscr{A}_{2} \subset E, \ldots, \mathscr{A}_{r} \subset E$.

(3) Obtain $r$ number q-ROFSSs on the $C M_{1}, C M_{2}, \ldots, C M_{r}$ respectively.

(4) Obtain curial opinions of $p$ number moderators on each q-ROFSSs. This formulate a GGq-ROFSSs $\left(\mathbb{Q}_{1}, \mathscr{A}_{1}, \eta_{1}\right),\left(\mathscr{Q}_{2}, \mathscr{A}_{2}, \eta_{2}\right), \ldots,\left(\mathbb{Q}_{r}, \mathscr{A}_{r}, \eta_{r}\right)$.

(5) Take union $\left(\mathscr{Q}_{1}, \mathscr{A}_{1}, \eta_{1}\right),\left(\mathscr{Q}_{2}, \mathscr{A}_{2}, \eta_{2}\right), \ldots,\left(\mathbb{Q}_{r}, \mathscr{A}_{r}, \eta_{r}\right)$ which is equal to $(\mathbb{Q}, \mathscr{A}, \eta)$, where $\mathscr{A}=\cup_{j^{\prime}=1}^{r} \mathscr{A}_{j^{\prime}}=\left\{e_{1}, e_{2}, \ldots, e_{n}\right\}$

(6) Normalize $(\mathbb{Q}, \mathscr{A}, \eta)$, which gives normalized GGq-ROFSS $\left(\mathscr{Q}^{\prime}, \mathscr{A}, \eta^{\prime}\right)$.

(7) Obtain the weighted vector $\Psi=\left[\varphi_{1}, \varphi_{2}, \ldots, \varphi_{n}\right]^{T}$ over set $A$ of attributes. Obtain the weighted vector $W=\left[\varpi_{0}, \emptyset_{1}, \oplus_{2}, \ldots, \emptyset_{p}\right]^{T}$ comprising q-ROFSS and opinions of moderators.

(8) Compute GGq-ROFA (or GGq-ROFG) operators $\Theta_{j}\left(\right.$ or $\left.\Theta_{j}^{\prime}\right)(j=1,2, \ldots, m)$ on normalized GGq-ROFSS $\left(Q^{\prime}, \mathscr{A}^{\prime}, \eta^{\prime}\right)$.

(9) Get score function $S_{\text {liu }}$ or $S_{p x}$ on each GGq-ROFA (or GGq-ROFG) operator $\Theta_{j}$ or $\Theta_{j}^{\prime}(j=1,2, \ldots, m$ ) using Definition 4.

(10) Classify alternatives and foremost alternative is on the maximum score.

Algorithm 1: MCDM procedure.

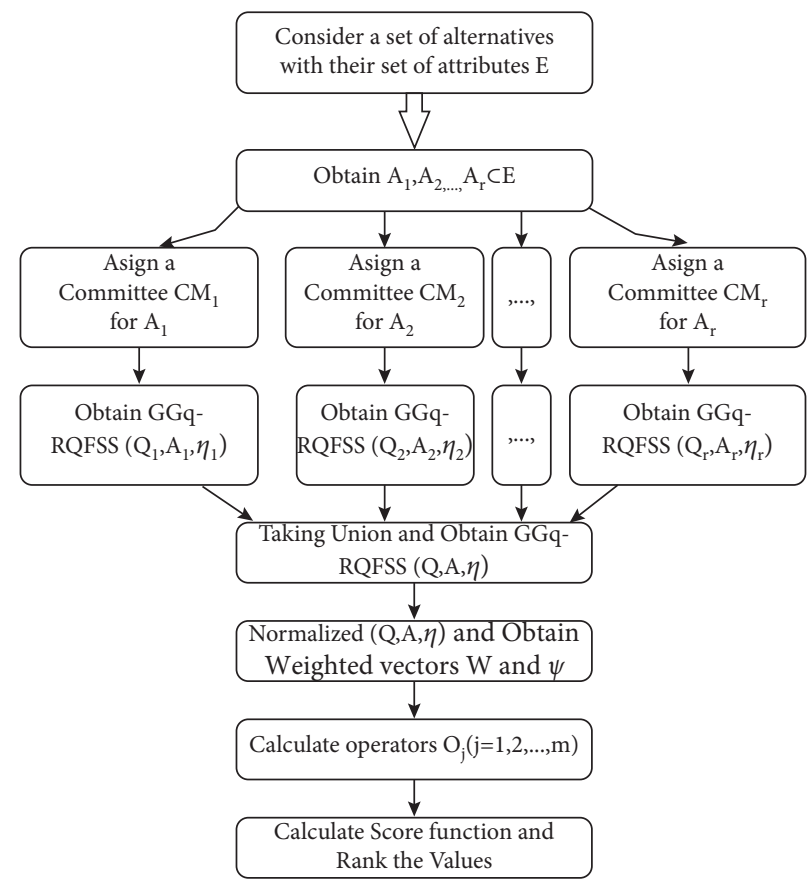

Figure 1: A flowchart of our algorithm.

Table 7: GGq-ROFSSs $(\mathscr{Q}, \mathscr{A}, \eta)$.

\begin{tabular}{cccc}
\hline$\chi \mid \mathscr{A}$ & $C_{1}$ & $C_{2}$ & $C_{5}$ \\
\hline$\rho_{1}$ & $\langle 0.7,0.7\rangle$ & $\langle 0.5,0.7\rangle$ & $\langle 0.6,0.8\rangle$ \\
$\rho_{2}$ & $\langle 0.8,0.5\rangle$ & $\langle 0.8,0.6\rangle$ & $\langle 0.6,0.7\rangle$ \\
$\rho_{3}$ & $\langle 0.5,0.4\rangle$ & $\langle 0.9,0.3\rangle$ & $\langle 0.7,0.4\rangle$ \\
$\rho_{4}$ & $\langle 0.5,0.7\rangle$ & $\langle 0.7,0.7\rangle$ & $\langle 0.7,0.8\rangle$ \\
\hline$\alpha_{1}$ & $\langle 0.6,0.7\rangle$ & $\langle 0.8,0.7\rangle$ & $\langle 0.6,0.5\rangle$ \\
$\alpha_{2}$ & $\langle 0.5,0.6\rangle$ & $\langle 0.6,0.5\rangle$ & $\langle 0.7,0.7\rangle$ \\
\hline
\end{tabular}

In order to evaluate most suitable candidate, two different committees of experts, namely, $C M_{1}$ and $C M_{2}$, are constituted by the company. The attribute specifications for $C M_{1}$ and $C M_{2}$ are $\mathscr{A}=\left\{C_{1}, C_{2}, C_{5}\right\}$ and $\mathscr{B}=\left\{C_{3}, C_{4}, C_{6}\right\}$,
TABle 8: GGq-ROFSSs $\left(\mathscr{Q} \prime, \mathscr{B}, \eta^{\prime}\right)$.

\begin{tabular}{cccc}
\hline$\chi \mid \mathscr{B}$ & $C_{3}$ & $C_{4}$ & $C_{6}$ \\
\hline$\rho_{1}$ & $\langle 0.6,0.3\rangle$ & $\langle 0.7,0.7\rangle$ & $\langle 0.8,0.4\rangle$ \\
$\rho_{2}$ & $\langle 0.8,0.4\rangle$ & $\langle 0.7,0.6\rangle$ & $\langle 0.8,0.7\rangle$ \\
$\rho_{3}$ & $\langle 0.9,0.3\rangle$ & $\langle 0.8,0.3\rangle$ & $\langle 0.7,0.5\rangle$ \\
$\rho_{4}$ & $\langle 0.6,0.7\rangle$ & $\langle 0.6,0.7\rangle$ & $\langle 0.5,0.6\rangle$ \\
\hline$\alpha_{1}^{\prime}$ & $\langle 0.4,0.3\rangle$ & $\langle 0.6,0.5\rangle$ & $\langle 0.7,0.7\rangle$ \\
$\alpha_{2}^{\prime}$ & $\langle 0.6,0.6\rangle$ & $\langle 0.7,0.5\rangle$ & $\langle 0.8,0.3\rangle$ \\
\hline
\end{tabular}

respectively. The $\mathrm{q}-\mathrm{ROFV}$-based evaluations are q-ROFSSs $(\mathscr{Q}, \mathscr{A})$ (in the light gray part of Table 7 ) and $(\mathscr{Q} \prime, \mathscr{B})$ (in the light gray part of Table 8 ). To finalize q-ROFV-based data, two senior experts provided their opinion as q-ROFSs on the 
TABle 9: GGq-ROFSSs $(\mathscr{R}, E, \gamma)$.

\begin{tabular}{ccccccc}
\hline$\chi \mid E$ & $C_{1}$ & $C_{2}$ & $C_{3}$ & $C_{4}$ & $C_{5}$ & $C_{6}$ \\
\hline$\rho_{1}$ & $\langle 0.7,0.7\rangle$ & $\langle 0.5,0.7\rangle$ & $\langle 0.6,0.3\rangle$ & $\langle 0.7,0.7\rangle$ & $\langle 0.6,0.8\rangle$ & $\langle 0.8,0.4\rangle$ \\
$\rho_{2}$ & $\langle 0.8,0.5\rangle$ & $\langle 0.8,0.6\rangle$ & $\langle 0.8,0.4\rangle$ & $\langle 0.7,0.6\rangle$ & $\langle 0.6,0.7\rangle$ & $\langle 0.8,0.7\rangle$ \\
$\rho_{3}$ & $\langle 0.5,0.4\rangle$ & $\langle 0.9,0.3\rangle$ & $\langle 0.9,0.3\rangle$ & $\langle 0.8,0.3\rangle$ & $\langle 0.7,0.4\rangle$ & $\langle 0.7,0.5\rangle$ \\
$\rho_{4}$ & $\langle 0.5,0.7\rangle$ & $\langle 0.7,0.7\rangle$ & $\langle 0.6,0.7\rangle$ & $\langle 0.6,0.7\rangle$ & $\langle 0.7,0.8\rangle$ & $\langle 0.5,0.6\rangle$ \\
\hline$\beta_{1}$ & $\langle 0.6,0.7\rangle$ & $\langle 0.8,0.7\rangle$ & $\langle 0.4,0.3\rangle$ & $\langle 0.6,0.5\rangle$ & $\langle 0.6,0.5\rangle$ & $\langle 0.7,0.7\rangle$ \\
$\beta_{2}$ & $\langle 0.5,0.6\rangle$ & $\langle 0.6,0.5\rangle$ & $\langle 0.6,0.6\rangle$ & $\langle 0.7,0.5\rangle$ & $\langle 0.7,0.7\rangle$ & $\langle 0.8,0.3\rangle$ \\
\hline
\end{tabular}

Table 10: GGq-ROFSS $(\mathscr{Q}, A, \eta)$.

\begin{tabular}{cccc}
\hline$\chi \mid \mathscr{A}$ & $e_{1}$ & $e_{2}$ & $e_{3}$ \\
\hline$\rho_{1}$ & $\langle 0.6,0.3\rangle$ & $\langle 0.5,0.4\rangle$ & $\langle 0.5,0.4\rangle$ \\
$\rho_{2}$ & $\langle 0.5,0.4\rangle$ & $\langle 0.4,0.3\rangle$ & $\langle 0.5,0.5\rangle$ \\
$\rho_{3}$ & $\langle 0.4,0.4\rangle$ & $\langle 0.6,0.3\rangle$ & $\langle 0.4,0.4\rangle$ \\
\hline$\alpha_{1}$ & $\langle 0.6,0.4\rangle$ & $\langle 0.3,0.4\rangle$ & $\langle 0.3,0.4\rangle$ \\
\hline
\end{tabular}

TABle 11: Comparison.

\begin{tabular}{lcc}
\hline Method & Act & Ranking \\
\hline Proposed method & $q=3$ & $\rho_{3}>\rho_{1}>\rho_{2}$ \\
Hayat et al. [55] & $q=1$ & $\rho_{3}>\rho_{1}>\rho_{2}$ \\
Hayat et al. [56] & $q=1$ & $\rho_{1}>\rho_{3}>\rho_{2}$ \\
Feng et al. [53] & $q=1$ & $\rho_{1}>\rho_{3}>\rho_{2}$ \\
Hayat et al. [59] & $q=1$ & $\rho_{3}>\rho_{1}>\rho_{2}$ \\
\hline
\end{tabular}

q-ROFSSs (see the brown part of Tables 7 and 8). The following steps are adopted to finalize decision-making process:

(1) Take union on GGq-ROFSSs $(\mathscr{Q}, \mathscr{A}, \eta)$ and $(\mathscr{Q}, \mathscr{B}$, $\left.\eta^{\prime}\right)$, and obtain GGq-ROFSS $(\mathscr{R}, E, \gamma)$ in Table 9.

(2) Take $\Psi=\left[\varpi_{1}, \oplus_{2}, \ldots, \oplus_{6}\right]^{T}=[0.2,0.3,0.1,0.2,0.1,0.1]^{T}$ be the weighted vector over $E$. Also take weighted vector $W=\left[\varphi_{0}, \varphi_{1}, \varphi_{2}\right]^{T}=[0.4,0.25,0.35]^{T} \quad$ for q-ROFSS and judgements of senior experts.

(3) Calculate GWq-ROFA operators; we use $q=4$ and obtain $\Theta_{1}=\langle 0.6490,0.5719\rangle, \Theta_{2}=\langle 0.7065,0.5555\rangle$, $\Theta_{3}=\langle 0.7431,0.4530\rangle$, and $\Theta_{4}=\langle 0.6464,0.6013\rangle$.

(4) Calculate score function; we obtain $S_{\text {liu }}\left(\Theta_{1}\right)=0.0771$, $S_{\text {liu }}\left(\Theta_{2}\right)=0.1510, S_{\text {liu }}\left(\Theta_{3}\right)=0.2901$, and $S_{\text {liu }}\left(\Theta_{4}\right)=$ 0.0451 .

(5) Final ranking of candidates is given by $\rho_{3}>\rho_{2}>\rho_{1}>$ $\rho_{4}$. Thus, $\rho_{3}$ is the most appropriate candidate for the position of director in the company.

\section{Comparative Study}

In this part of the article, we compare our method with existing frameworks. The primely advantage of new
TABle 12: Advantages.

\begin{tabular}{|c|c|c|c|c|}
\hline $\begin{array}{l}\text { Methods } \backslash \\
\text { approches }\end{array}$ & $\begin{array}{l}\text { Numbers } \\
\text { of extra } \\
\text { inputs }\end{array}$ & $\begin{array}{l}\text { Weighted } \\
\text { vectors }\end{array}$ & $\begin{array}{c}\text { Operators } \\
\text { type }\end{array}$ & Deficiency \\
\hline $\begin{array}{l}\text { Hayat } \\
\text { et al. [55] }\end{array}$ & One & None & $\begin{array}{c}\text { AND } \\
\text { operation }\end{array}$ & $\begin{array}{c}\text { Diminish the } \\
\text { importance } \\
\text { of reliable } \\
\text { and } \\
\text { unreliable } \\
\text { grades on } \\
\text { AND } \\
\text { operation }\end{array}$ \\
\hline $\begin{array}{l}\text { Hayat } \\
\text { et al. [56] }\end{array}$ & $\begin{array}{l}\text { More than } \\
\text { one }\end{array}$ & $\begin{array}{c}\text { Over } \\
\text { attributes }\end{array}$ & $\begin{array}{c}\text { WA or } \\
\text { WG }\end{array}$ & $\begin{array}{l}\text { Not efficient } \\
\text { on the same } \\
\text { IF values of a } \\
\text { moderator } \\
\text { on each } \\
\text { attribute }\end{array}$ \\
\hline $\begin{array}{l}\text { Hayat } \\
\text { et al. [59] }\end{array}$ & $\begin{array}{l}\text { More than } \\
\text { one }\end{array}$ & $\begin{array}{c}\text { Two } \\
\text { weighted } \\
\text { vectors over } \\
\text { attributes } \\
\text { and } \\
\text { moderators } \\
\text { respectively }\end{array}$ & $\begin{array}{c}\text { WA or } \\
\text { WG }\end{array}$ & $\begin{array}{l}\text { Do not deal } \\
\text { with larger } \\
\text { space } q>1\end{array}$ \\
\hline $\begin{array}{l}\text { Feng et al. } \\
\text { [53] }\end{array}$ & One & $\begin{array}{l}\text { Conversion } \\
\text { of extra input } \\
\text { into } \\
\text { weighted } \\
\text { vector }\end{array}$ & $\begin{array}{c}\text { WA or } \\
\text { WG }\end{array}$ & $\begin{array}{l}\text { Diminish the } \\
\text { importance } \\
\text { of reliable } \\
\text { and } \\
\text { unreliable } \\
\text { grades by the } \\
\text { conversion } \\
\text { of extra input } \\
\text { into } \\
\text { weighted } \\
\text { vector }\end{array}$ \\
\hline $\begin{array}{l}\text { Proposed } \\
\text { method }\end{array}$ & $\begin{array}{l}\text { More than } \\
\text { one }\end{array}$ & $\begin{array}{c}\text { Two } \\
\text { weighted } \\
\text { vectors over } \\
\text { attributes } \\
\text { and } \\
\text { moderators }\end{array}$ & $\begin{array}{c}\text { WA or } \\
\text { WG }\end{array}$ & $\begin{array}{l}\text { Seem no } \\
\text { deficiency }\end{array}$ \\
\hline
\end{tabular}

aggregation operators is to characterize a weighted vector $W=\left[\varphi_{0}, \varphi_{1}, \varphi_{2}, \ldots, \varphi_{p}\right]^{T}$ on GGq-ROFSS, where $\varphi_{1}, \varphi_{2}, \ldots$, $\varphi_{p}$ are the weights on q-ROFSs of $p$ number of moderators respectively, and $\varphi_{0}$ is a weight for q-ROFSS in GGq-ROFSS. Since assessments of moderators on q-ROFSS are the crucial component in GGq-ROFSS, in many real-life problems, their weights should be greater than $\varphi_{0}$. On the other hand, in some real-life situations, weight $\varphi_{0}$ on q-ROFSS is also important, the fact that it denotes a perception on fundamental data (or q-ROFSS) given by a committee of experts on crucial judgements on alternatives. Notably, we consider following example.

Example 4. Consider GGq-ROFSS as depicted in Table 6, where $\mathscr{A}=\left\{e_{1}, e_{2}, e_{3}\right\}$ is set of attributes with weighted vector $\Psi=\left\{\left(0.3 / e_{1}\right),\left(0.4 / e_{2}\right),\left(0.3 / e_{3}\right)\right\}$ and $\mathscr{X}=\left\{\rho_{1}, \rho_{2}, \rho_{3}\right\}$ being the set of alternatives. $\alpha_{1}$ is q-ROFS for moderator's assessments on q-ROFSS in Table 10. 
Let $q=3$ and a weighted vector $W=\left[\left(0.5 / w_{0}\right),(0.5 /\right.$ $\left.w_{1}\right)$ ], where $w_{0}$ is the weight for q-ROFSS and $w_{1}$ is the weight for q-ROFS $\alpha_{1}$. By our new operators on GGqROFSSs, we obtain $S_{\text {liu }}\left(\Theta_{1}\right)=0.0233, S_{\text {liu }}\left(\Theta_{2}\right)=0.0225$, $S_{\text {liu }}\left(\Theta_{3}\right)=0.0235$ and $\rho_{3}>\rho_{1}>\rho_{2}$.

By the operators in Hayat et al. [56], we obtain $S\left(\rho_{1}\right)=$ 0.7883, $S\left(\rho_{2}\right)=0.7646, S\left(\rho_{3}\right)=0.7777$ and $\rho_{1}>\rho_{3}>\rho_{2}$.

By Feng et al. [54], $S\left(\rho_{1}\right)=0.5931, S\left(\rho_{2}\right)=0.5398$, $S\left(\rho_{3}\right)=0.5509, \rho_{1}>\rho_{3}>\rho_{2}$.

By the operators in Hayat et al. [55, 59], we obtain $\rho_{1}>\rho_{3}>\rho_{2}$. A comparison for different methods on this example is shown in Table 11.

The main advantage our framework is that it aggregates information or data by concerning attributes until final ranking appears. It develop an internal mechanism that gently addresses the importance of parameters in aggregation operators for GGq-ROFSSs. The different components of the above methods are discussed in Table 12.

\section{Conclusions}

In this article, we have presented GGq-ROFSS and investigated some basic operations. Mainly, we have initiated new averaging and geometric aggregation operators on GGqROFSSs and investigated the underlying properties of these operators. We have given a MCDM framework and its validation through a numerical example. Finally, we have given comparison of our methodology with other existing methods. In GGq-ROFSSs, expert's judgements suggest the reliability of the evaluation of the alternatives on criteria. These judgements appear as generalization part in GGqROFSSs to fulfil and complete q-ROFSS-based data as some final examination. Therefore, for prospect MCDM, GGqROFSS, which indicates in $\mu^{q}+\nu^{q} \leq 1$ for some $q \geq 1$ suggests a better framework to deal with uncertainty.

The method of computation of q-ROFS-based data from newly introduced ideas can sight wide applications for available data in machine learning, applied intelligence, and supply chain management. Although there are some methods in machine learning, artificial intelligence, and supply chain management, proposed operators' beauty will be a notable sight in such domains. Our focus will be on these operators' new rudiments and their supply chain management applications in future work. Furthermore, we will define GGq-ROFSS-based order operators, Shapley Choquet operators, and distance aggregation operator.

\section{Data Availability}

No data were used to support the study.

\section{Conflicts of Interest}

The authors declare no conflicts of interest.

\section{Acknowledgments}

This research was supported by the Researchers Supporting Project number (RSP-2021/244), King Saud University, Riyadh, Saudi Arabia.

\section{References}

[1] L. A. Zadeh, "Fuzzy sets," Information and Control, vol. 8, no. 3, pp. 338-353, 1965.

[2] K. T. Atanassov, "Intuitionistic fuzzy sets," Fuzzy Sets and Systems, vol. 20, no. 1, pp. 87-96, 1986.

[3] Z. Xu and R. R. Yager, "Some geometric aggregation operators based on intuitionistic fuzzy sets," International Journal of General Systems, vol. 35, no. 4, pp. 417-433, 2006.

[4] Z. S. Xu, "Intuitionistic fuzzy aggregation operators," IEEE Transactions on Fuzzy Systems, vol. 15, no. 6, pp. 1179-1187, 2007.

[5] E. Szmidt and J. Kacprzyk, "Distances between intuitionistic fuzzy sets," Fuzzy Sets and Systems, vol. 114, no. 3, pp. 505-518, 2000.

[6] P. Milosevic, B. Petrovic, and V. Jeremic, "IFS-IBA similarity measure in machine learning algorithms," Expert Systems with Applications, vol. 89, pp. 296-305, 2017.

[7] X. Zhang, P. Liu, and Y. Wang, "Multiple attribute group decision making methods based on intuitionistic fuzzy frank power aggregation operators," Journal of Intelligent \& Fuzzy Systems, vol. 29, no. 5, pp. 2235-2246, 2015.

[8] M. Lin, W. Xu, Z. Lin, and R. Chen, "Determine OWA operator weights using kernel density estimation," Economic Research-Ekonomska Istraživanja, vol. 33, no. 1, pp. 1441-1464, 2020.

[9] M. Qiyas, M. A. Khan, S. Khan, and S. Abdullah, "Concept of Yager operators with the picture fuzzy set environment and its application to emergency program selection," International Journal of Intelligent Computing and Cybernetics, vol. 13, 2020.

[10] X. J. Gou, Z. Xu, and Q. Lei, "New operational laws and aggregation method of intuitionistic fuzzy information," Journal of Intelligent \& Fuzzy Systems, vol. 30, pp. 129-141, 2016.

[11] Z. Xu and N. Zhao, "Information fusion for intuitionistic fuzzy decision making: an overview," Information Fusion, vol. 28, pp. 10-23, 2016.

[12] R. R. Yager, "Pythagorean fuzzy subsets," in Proceedings of the Joint IFSA World Congress and NAFIPS Annual Meeting, pp. 57-61, Edmonton, Canada, 2013.

[13] R. R. Yager, "Pythagorean membership grades in multicriteria decision making," IEEE Transactions on Fuzzy Systems, vol. 22, no. 4, pp. 958-965, 2014.

[14] R. R. Yager, "Generalized orthopair fuzzy sets," IEEE Transactions on Fuzzy Systems, vol. 25, pp. 1222-1230, 2016.

[15] M. I. Ali, "Another view on q-rung orthopair fuzzy sets," International Journal of Intelligent Systems, vol. 33, no. 11, pp. 2139-2153, 2018.

[16] P. Liu and P. Wang, "Some q-rung orthopair fuzzy aggregation operators and their applications to multiple-attribute decision making," International Journal of Intelligent Systems, vol. 33, no. 2, pp. 259-280, 2018.

[17] M. Lin, C. Huang, R. Chen, H. Fujita, and X. Wang, "Directional correlation coefficient measures for Pythagorean fuzzy sets; their applications to medical diagnosis and cluster analysis," Complex \& Intelligent Systems, vol. 7, pp. 10251043, 2021.

[18] X. Gou, Z. Xu, and P. Ren, "The properties of continuous Pythagorean fuzzy information," International Journal of Intelligent Systems, vol. 31, no. 5, pp. 401-424, 2016.

[19] J. Gao and Z. Xu, "Differential calculus of interval-valued q-rung orthopair fuzzy functions and their applications," International Journal of Intelligent Systems, vol. 34, no. 12, pp. 3190-3219, 2019. 
[20] R. R. Yager, "Extending set measures to pythagorean fuzzy sets," International Journal of Fuzzy Systems, vol. 21, no. 2, pp. 343-354, 2019.

[21] X. Peng and Z. Luo, "A review of q-rung orthopair fuzzy information: bibliometrics and future directions," Artificial Intelligence Review, vol. 54, pp. 3361-3430, 2021.

[22] P. Ren, Z. Xu, and X. Gou, "Pythagorean fuzzy TODIM approach to multi-criteria decision making," Applied Soft Computing, vol. 42, pp. 246-259, 2016.

[23] X. Zhang and Z. Xu, "Extension of TOPSIS to multiple criteria decision making with Pythagorean fuzzy sets," International Journal of Intelligent Systems, vol. 29, no. 12, pp. 1061-1078, 2014.

[24] N. Alkan and C. Kahraman, "Evaluation of government strategies against COVID-19 pandemic using q-rung orthopair fuzzy TOPSIS method," Applied Soft Computing, vol. 110, Article ID 107653, 2021.

[25] H. Li, L. Lv, F. Li, L. Wang, and Q. Xia, "A novel approach to emergency risk assessment using FMEA with extended MULTIMOORA method under interval valued Pythagorean fuzzy environment," International Journal of Intelligent Computing and Cybernetics, vol. 13, 2020.

[26] J. Wang, G. Wei, C. Wei, and Y. Wei, "MABAC method for multiple attribute group decision making under q-rung orthopair fuzzy environment," Defence Technology, vol. 16, no. 1, pp. 208-216, 2020.

[27] P. Liu, P. Liu, P. Wang, and B. Zhu, “An extended multiple attribute group decision making method based on q-rung orthopair fuzzy numbers," IEEE Access, vol. 7, pp. 162050162061, 2019.

[28] P. Liu, Z. Ali, and T. Mahmood, "A method to multi-attribute group decision-making problem with complex q-rung orthopair linguistic information based on heronian mean operators," International Journal of Computational Intelligence Systems, vol. 12, no. 2, pp. 1465-1496, 2019.

[29] Y. Qin, X. Cui, M. Huang, Y. Zhong, Z. Tang, and P. Shi, "Archimedean Muirhead aggregation operators of q-rung orthopair fuzzy numbers for multicriteria group decision making," Complexity, vol. 2019, Article ID 3103741, 33 pages, 2019.

[30] P. Liu and Y. Wang, "Multiple attribute decision making based on q-rung orthopair fuzzy generalized Maclaurin symmetic mean operators," Information Sciences, vol. 518, pp. 181-210, 2020.

[31] M. Lin, X. Li, and L. Chen, "Linguistic q-rung orthopair fuzzy sets and their interactional partitioned Heronian mean aggregation operators," International Journal of Intelligent Systems, vol. 35, no. 2, pp. 217-249, 2020.

[32] X. Peng, J. Dai, and H. Garg, "Exponential operation and aggregation operator for q-rung orthopair fuzzy set and their decision-making method with a new score function," International Journal of Intelligent Systems, vol. 33, no. 11, pp. 2255-2282, 2018.

[33] L. Wang and H. Garg, "Algorithm for multiple attribute decision-making with interactive archimedean norm operations under Pythagorean fuzzy uncertainty," International Journal of Computational Intelligence Systems, vol. 14, pp. 503-527, 2021.

[34] R. Verma, "Multiple attribute group decision-making based on order- $\alpha$ divergence and entropy measures under q-rung orthopair fuzzy environment," International Journal of Intelligent Systems, vol. 35, no. 4, pp. 718-750, 2020.

[35] S. Zeng, Y. Hu, and X. Xie, "Q-rung orthopair fuzzy weighted induced logarithmic distance measures and their application in multiple attribute decision making," Engineering Applications of Artificial Intelligence, vol. 100, Article ID 104167, 2021.

[36] D. Molodtsov, "Soft set theory-first results," Computers \& Mathematics with Applications, vol. 37, no. 4-5, pp. 19-31, 1999.

[37] P. K. Maji, R. Biswas, and A. R. Roy, "Soft set theory," Computers \& Mathematics with Applications, vol. 45, no. 4-5, pp. 555-562, 2003.

[38] M. I. Ali, F. Feng, X. Liu, W. K. Min, and M. Shabir, "On some new operations in soft set theory," Computers \& Mathematics with Applications, vol. 57, no. 9, pp. 1547-1553, 2009.

[39] P. K. Maji, R. Biswas, and A. R. Roy, "Fuzzy soft sets," Journal of Fuzzy Mathematics, vol. 9, no. 3, pp. 589-602, 2001.

[40] U. Acar, F. Koyuncu, and B. Tanay, "Soft sets and soft rings," Computers \& Mathematics with Applications, vol. 59, no. 11, pp. 3458-3463, 2010.

[41] J. C. R. Alcantud, "Some formal relationships among soft sets, fuzzy sets, and their extensions," International Journal of Approximate Reasoning, vol. 68, pp. 45-53, 2016.

[42] J. C. R. Alcantud, "A novel algorithm for fuzzy soft set based decision making from multiobserver input parameter data set," Information Fusion, vol. 29, pp. 142-148, 2016.

[43] F. Feng, H. Fujita, Y. B. Jun, and M. Khan, "Decomposition of fuzzy soft sets with finite value spaces," The Scientific World Journal, vol. 2014, Article ID 902687, 10 pages, 2014.

[44] M. Akram, F. Feng, A. B. Saeid, and V. Leoreanu-Fotea, "A new multiple criteria decision-making method based on bipolar fuzzy soft graphs," Iranian Journal of Fuzzy Systems, vol. 15, pp. 73-92, 2018.

[45] Y. Liu, K. Qin, and L. Martínez, "Improving decision making approaches based on fuzzy soft sets and rough soft sets," Applied Soft Computing, vol. 65, pp. 320-332, 2018.

[46] M. Akram, N. O. Al-Shehrie, K. P. Shum, and A. Farooq, "Application of bipolar fuzzy soft sets in K-algebras," Italian Journal of Pure and Applied Mathematics, vol. 32, pp. 1-14, 2014.

[47] P. K. Maji, R. Biswas, and A. R. Roy, "Intuitionistic fuzzy soft sets," Journal of Fuzzy Mathematics, vol. 9, no. 3, pp. 677-692, 2001.

[48] M. T. Hamid, M. Riaz, and D. Afzal, "Novel MCGDM with q-rung orthopair fuzzy soft sets and TOPSIS approach under q-rung orthopair fuzzy soft topology," Journal of Intelligent and Fuzzy Systems, vol. 39, 2020.

[49] M. Riaz, M. T. Hamid, H. M. A. Farid, and D. Afzal, "TOPSIS, VIKOR and aggregation operators based on q-ROFSS," Journal of Intelligent and Fuzzy Systems, vol. 39, no. 5, pp. 6903-6917, 2020.

[50] A. Hussain, M. I. Ali, T. Mahmood, and M. Munir, "q-Rung orthopair fuzzy soft average aggregation operators and their application in multicriteria decision-making," International Journal of Intelligent Systems, vol. 35, no. 4, pp. 571-599, 2020.

[51] M. Agarwal, K. K. Biswas, and M. Hanmandlu, "Generalized intuitionistic fuzzy soft sets with applications in decisionmaking," Applied Soft Computing, vol. 13, no. 8, pp. 3552-3566, 2013.

[52] A. M. Khalil, "Commentary on "generalized intuitionistic fuzzy soft sets with applications in decision-making", "Applied Soft Computing, vol. 37, pp. 519-520, 2015.

[53] F. Feng, H. Fujita, M. I. Ali, R. R. Yager, and X. Liu, "Another View on generalized intuitionistic fuzzy soft sets and related multiattribute decision making methods," IEEE Transactions on Fuzzy Systems, vol. 27, pp. 474-488, 2018. 
[54] H. Garg and R. Arora, "Generalized and group-based generalized intuitionistic fuzzy soft sets with applications in decision-making," Applied Intelligence, vol. 48, no. 2, pp. 343-356, 2018.

[55] K. Hayat, M. I. Ali, J. C. R. Alcantud, B. Y. Cao, and K. U. Tariq, "Best concept selection in design process: an application of generalized intuitionistic fuzzy soft sets," Journal of Intelligent \& Fuzzy Systems, vol. 35, pp. 1-14, 2018.

[56] K. Hayat, M. I. Ali, B. Y. Cao, F. Karaaslan, and X. P. Yang, "Another view of aggregation operators on group-based generalized intuitionistic fuzzy soft sets: multiattribute decision making method," Symmetry, vol. 10, pp. 1-18, 2018.

[57] M. J. Khan, P. Kuman, P. Liu, and W. Kuman, "An adjustable weighted soft discernibility matrix based on generalized picture fuzzy soft set and its applications in decision making," Journal of Intelligent \& Fuzzy Systems, vol. 43, pp. 1-16, 2020.

[58] M. J. Khan, P. Kuman, P. Liu, and W. Kuman, "Another view on generalized interval valued intuitionistic fuzzy soft set and its applications in decision support system," Journal of Intelligent \& Fuzzy Systems, vol. 43, pp. 1-15, 2020.

[59] K. Hayat, Z. Tariq, E. Lughofer, and M. F. Aslam, "New aggregation operators on group-based generalized intuitionistic fuzzy soft sets," Soft Computing, vol. 25, pp. 1335313364, 2021. 